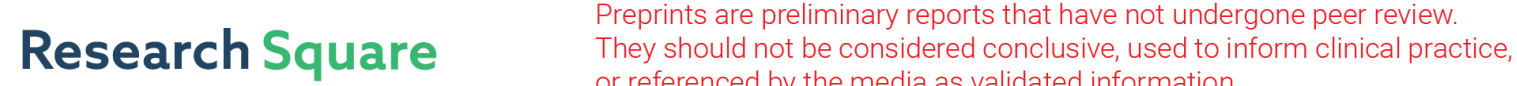 or referenced by the media as validated information. \\ Stabilization of Energy Level Sets of Underactuated Mechanical Systems Exploiting Impulsive Braking
}

\author{
Nilay Kant \\ Michigan State University College of Engineering \\ Ranjan Mukherjee ( $\square$ mukherj@@egr.msu.edu ) \\ Michigan State University https://orcid.org/0000-0002-7211-1362 \\ Hassan K Khalil \\ Michigan State University
}

\section{Research Article}

Keywords: energy level set, impulsive dynamics, underactuated mechanical systems

Posted Date: May 4th, 2021

DOI: https://doi.org/10.21203/rs.3.rs-445534/v1

License: (1) This work is licensed under a Creative Commons Attribution 4.0 International License.

Read Full License 


\title{
Stabilization of Energy Level Sets of Underactuated Mechanical Systems Exploiting Impulsive Braking
}

\author{
Nilay Kant · Ranjan Mukherjee · Hassan Khalil
}

\begin{abstract}
Recent investigations of underactuated systems have demonstrated the benefits of control inputs that are impulsive in nature. Here we consider the problem of stabilization of energy level sets of underactuated systems exploiting impulsive braking. We consider systems with one passive degree-of-freedom (DOF) and the energy level set is a manifold where the active coordinates are fixed and the mechanical energy equals some desired value. A control strategy comprised of continuous inputs and intermittent impulsive braking inputs is presented. The generality of the approach is shown through simulation of a three-DOF Tiptoebot; the feasibility of implementation of impulsive control using standard hardware is demonstrated using a rotary pendulum.
\end{abstract}

Keywords energy level set · impulsive dynamics . underactuated mechanical systems

\section{INTRODUCTION}

In many applications, such as legged locomotion [1,2], underactuated systems are required to undergo repetitive motion and orbital stabilization is the control objective. To achieve repetitive motion, geometric con-

N. Kant

Mechanical Engineering Dept., Michigan State University, MI 48824, USA

E-mail: kantnila@msu.edu

R. Mukherjee

Mechanical Engineering Dept., Michigan State University, MI 48824, USA

E-mail: mukherji@egr.msu.edu

Hassan Khalil

Electrical Engineering Dept., Michigan State University, MI 48824, USA

E-mail: khalil@msu.edu straints are imposed on the generalized coordinates using the virtual holonomic constraint (VHC) approach [3]6]. Orbital stabilization has also been used for swingup control of underactuated systems with one passive degree-of-freedom (DOF). Some examples include twoDOF systems such as the pendubot [7, the acrobot [8], the reaction-wheel pendulum 9], inverted pendulum on a cart [10,11, the rotary pendulum [12, and the threeDOF gymnast robot [13. These controllers stabilize an energy level set that include the equilibrium, which is typically unstable. Unlike the VHC approach, geometric constraints are not imposed; instead, the controllers are designed to pump energy in and out of the system and converge the active DOFs to their desired configuration. Such control designs are typically based on a Lyapunov-like function that is comprised of terms involving positions and velocities of the active DOFs and the total mechanical energy of the system. Although the structure of the Lyapunov-like function is identical, the stability analysis is different for each system due to the difference in the nature of their nonlinear dynamics. Despite the effectiveness of the individual controllers, a general methodology for stabilizing an energy level set does not exist. In this paper, we present a control strategy for n-DOF underactuated systems with one passive DOF based on continuous-time inputs and intermittent impulsive braking inputs 1 .

Prior works on impulsive control [15 21] have been theoretical in nature but in recent works [6,22, 29, impulsive inputs have been utilized for control of underactuated systems in both simulations and experiments. In experiments, impulsive inputs have been implemented in standard hardware using high-gain feedback [23,24, dispelling the notion that impulsive inputs require large

\footnotetext{
1 Impulsive braking for control of the underctuated systems was first proposed in [14] for swing-up of the pendubot.
} 
actuators and are impractical. A combination of continuous and impulsive inputs was used recently for stabilization of homoclinic orbits of two-DOF underactuated systems [27. This work provides a generalization of the theory to $\mathrm{n}-\mathrm{DOF}$ systems and experimental validation.

In this paper, we present a control design for stabilizing an energy level set for underactuated systems with one passive revolute joint. The energy level set is defined by fixed positions of the active coordinates and a desired mechanical energy of the system. The controller is comprised of continuous-time inputs and impulsive braking inputs. At first, a general result for underactuated systems is presented which shows that an impulsive input causes an instantaneous jump in the energy of the system; this jump is shown to be explicitly dependent on the change in the active velocities. This result is then used to show that impulsive braking causes a negative jump in the energy of the system as well as in a Lyapunov-like function. Finally, using a state-dependent impulsive dynamical system model [16], we present sufficient conditions for stabilization. To demonstrate the generality of our approach, we demonstrate stabilization of energy level sets for the three-DOF Tiptoebot 24] through simulations. Experimental validation is carried out on a rotary pendulum to show the applicability of our approach in real hardware. The main contributions of this work are as follows:

1. Our control design is applicable to a class of underactuated systems; a majority of underactuated systems investigated in the literature belong to this class.

2. The stability analysis is presented for the general case and it results in sufficient conditions that are not restrictive and can be verified.

3. Experimental validation is provided.

4. Impulsive braking is accomplished using a friction brake; this eliminates the need for high-gain feedback [23] which may result in actuator saturation.

\section{Problem Statement}

Consider an $n$-DOF underactuated system with one passive DOF. The generalized coordinates of the system are denoted by $q, q \triangleq\left[\begin{array}{ll}q_{1}^{T} & q_{2}\end{array}\right]^{T}$, where $q_{1} \in R^{n-1}$ and $q_{2} \in R$ are the coordinates associated with the active and passive DOFs. Our control objective is to stabilize the orbit defined by

$\left(q_{1}, \dot{q}_{1}, E\right)=\left(0,0, E_{\mathrm{des}}\right)$

where $E$ is the total mechanical energy of the system and is given by the relation

$E(q, \dot{q})=\frac{1}{2} \dot{q}^{T} M(q) \dot{q}+\mathcal{F}(q)$ and $E_{\text {des }}$ is the desired value of $E$. In (2), $M(q) \in R^{n \times n}$ is the mass matrix, assumed to be positive definite, and $\mathcal{F}(q)$ is the potential energy, assumed to be a smooth function of $q$. The mass matrix is partitioned as

$M(q)=\left[\begin{array}{l|l}M_{11}(q) & M_{12}(q) \\ \hline M_{12}^{T}(q) & M_{22}(q)\end{array}\right]$

where $M_{11} \in R^{(n-1) \times(n-1)}$ and $M_{22} \in R$.

Assumption 1 The energy of the system is periodic in the passive coordinate $q_{2}$, such that $E\left(q_{2}+2 \pi\right)=E\left(q_{2}\right)$.

Remark 1 Assumption 1 is easily satisfied if the passive $\mathrm{DOF}$ is a revolute joint.

Assumption 2 The elements of the mass matrix $M(q)$ are bounded and the potential energy $\mathcal{F}(q)$ is lower bounded.

Remark 2 The boundedness property of $M(q)$ and $\mathcal{F}(q)$ is satisfied for systems that have no prismatic joints.

\section{Modeling of System Dynamics}

\subsection{Euler-Lagrange Equations}

For our system described in section 2, the equations of motion can be written as:

$\frac{d}{d t}\left(\frac{\partial \mathcal{L}}{\partial \dot{q}_{1}}\right)-\left(\frac{\partial \mathcal{L}}{\partial q_{1}}\right)=u$

$\frac{d}{d t}\left(\frac{\partial \mathcal{L}}{\partial \dot{q}_{2}}\right)-\left(\frac{\partial \mathcal{L}}{\partial q_{2}}\right)=0$

where $\mathcal{L}(q, \dot{q})$ is the Lagrangian and $u \in R^{n-1}$ is the vector of independent control inputs. Each element of the vector $u$ is a continuous function of time for all $t \geq 0$ except at discrete instants $t=\tau_{k}, k=1,2, \cdots$, where it is impulsive in nature. At $t=\tau_{k}$, the impulsive input vector has the form $u\left(\tau_{k}\right)=\mathcal{I}_{k} \delta\left(t-\tau_{k}\right)$, where $\delta\left(t-\tau_{k}\right)$ is the Dirac measure at time $\tau_{k}$ and $\mathcal{I}_{k} \in R^{n-1}$ is the impulse of the impulsive input. The Lagrangian is

$\mathcal{L}(q, \dot{q})=\frac{1}{2} \dot{q}^{T} M(q) \dot{q}-\mathcal{F}(q)$

By substituting (3) in (5), the Lagrangian is written as

$\mathcal{L}(q, \dot{q})=\frac{1}{2} \dot{q}_{1}^{T} M_{11} \dot{q}_{1}+\frac{1}{2} M_{22} \dot{q}_{2}^{2}+\dot{q}_{1}^{T} M_{12} \dot{q}_{2}-\mathcal{F}$

and by substituting (6) in (4), the equations of motion become:

$M_{11} \ddot{q}_{1}+M_{12} \ddot{q}_{2}+h_{1}(q, \dot{q})=u$
$M_{12}^{T} \ddot{q}_{1}+M_{22} \ddot{q}_{2}+h_{2}(q, \dot{q})=0$ 
where

$$
\begin{aligned}
h_{1} & =\dot{M}_{11} \dot{q}_{1}+\dot{M}_{12} \dot{q}_{2}-\frac{1}{2}\left[\frac{\partial}{\partial q_{1}}\left(M_{11} \dot{q}_{1}\right)\right] \dot{q}_{1} \\
& -\left[\frac{\partial\left(M_{12} \dot{q}_{2}\right)}{\partial q_{1}}\right] \dot{q}_{1}-\frac{1}{2}\left[\frac{\partial M_{22}}{\partial q_{1}}\right]^{T} \dot{q}_{2}^{2}+\left[\frac{\partial \mathcal{F}}{\partial q_{1}}\right]^{T} \\
h_{2} & =\dot{M}_{22} \dot{q}_{2}+\dot{q}_{1}^{T} \dot{M}_{12}-\frac{1}{2} \dot{q}_{1}^{T}\left[\frac{\partial\left(M_{11} \dot{q}_{1}\right)}{\partial q_{2}}\right] \\
& -\frac{1}{2}\left[\frac{\partial M_{22}}{\partial q_{2}}\right] \dot{q}_{2}^{2}-\dot{q}_{1}^{T}\left[\frac{\partial\left(M_{12} \dot{q}_{2}\right)}{\partial q_{1}}\right]+\frac{\partial \mathcal{F}}{\partial q_{2}}
\end{aligned}
$$

Equations (7a) and (7b) can be rewritten in the form

$\ddot{q}_{1}=A(q, \dot{q})+B(q) u$

$\ddot{q}_{2}=-\left(1 / M_{22}\right)\left[M_{12}^{T}\{A(q, \dot{q})+B(q) u\}+h_{2}\right]$

where

$$
\begin{aligned}
B(q) & =\left[M_{11}-\left(1 / M_{22}\right) M_{12} M_{12}^{T}\right]^{-1} \\
A(q, \dot{q}) & =\left(1 / M_{22}\right) B(q)\left[M_{12} h_{2}-h_{1} M_{22}\right]
\end{aligned}
$$

Using properties of the mass matrix $M(q)$ and the Schur complement theorem [30, it can be shown that $B(q)$ is symmetric and positive-definite, i.e., $B(q)=B^{T}(q)>$ 0 .

\subsection{Effect of Impulsive Inputs}

When the input $u$ in (7a) is impulsive, it causes discontinuous jumps in the velocities $\left(\dot{q}_{1}, \dot{q}_{2}\right)$, while the coordinates $\left(q_{1}, q_{2}\right)$ remain unchanged. For the impulsive input at $t=\tau_{k}$, the jump in the velocities is computed by integrating (7) as follows 31]

$\left[\begin{array}{ll}M_{11} & M_{12} \\ M_{12}^{T} & M_{22}\end{array}\right]\left[\begin{array}{c}\Delta \dot{q}_{1} \\ \Delta \dot{q}_{2}\end{array}\right]=\left[\begin{array}{c}\mathcal{I}_{k} \\ 0\end{array}\right], \quad \mathcal{I}_{k} \triangleq \int_{t_{k}^{-}}^{t_{k}^{+}} u\left(t_{k}\right) d t$

In the above equation, $\Delta \dot{q}_{1}$ and $\Delta \dot{q}_{2}$ are defined as

$\Delta \dot{q}_{1} \triangleq\left(\dot{q}_{1}^{+}-\dot{q}_{1}^{-}\right), \quad \Delta \dot{q}_{2} \triangleq\left(\dot{q}_{2}^{+}-\dot{q}_{2}^{-}\right)$

where $\dot{q}^{-} \triangleq \dot{q}\left(\tau_{k}^{-}\right)$and $\dot{q}^{+} \triangleq \dot{q}\left(\tau_{k}^{+}\right)$denote the generalized velocities immediately before and after application of the impulsive inputs. Since the system is underactuated, the jump in $\dot{q}_{2}$ is dependent on the jumps in $\dot{q}_{1}$; this dependence is described by the one-dimensional impulse manifold [23] or impulse line, obtained from the equation above:

$\dot{q}_{2}^{+}=\dot{q}_{2}^{-}-\left(1 / M_{22}\right) M_{12}^{T}\left(\dot{q}_{1}^{+}-\dot{q}_{1}^{-}\right)$

The kinetic energy undergoes an instantaneous change due to jumps in the generalized velocities. This change is also equal to the change in the total mechanical energy of the system since the potential energy is only a function of the generalized coordinates. A formal statement of this result is provided next.
Lemma 1 For the dynamical system in (7), the jump in the total mechanical energy due to application of an impulsive input is given by

$\Delta E \triangleq\left(E^{+}-E^{-}\right)=\frac{1}{2} \dot{q}_{1}^{+^{T}} B^{-1}(q) \dot{q}_{1}^{+}-\frac{1}{2} \dot{q}_{1}^{-{ }^{T}} B^{-1}(q) \dot{q}_{1}^{-}$

where $E^{-}$and $E^{+}$are the energies immediately before and after application of the impulsive input.

Proof: See section 8.1 of Appendix.

Remark 3 The proof of Lemma 1 is provided for the general case where the number of active and passive DOFs are $(n-m)$ and $m$, respectively. This general result indicates that the change in mechanical energy due to an impulsive input depends only on the velocities of the active DOFs immediately before and after application of the input. The result is analogous to the passivity property for the continuous-time case [32, where the power input to the system is the inner product of the velocities of the active DOFs and control inputs. It is important to note that results similar to Lemma 1 appeared earlier in 33.

Impulsive braking results in $\dot{q}_{1}^{+}=0$. Thus it follows from Lemma 1 that impulsive braking results in a loss of mechanical energy, given by the expression

$\Delta E=-\frac{1}{2} \dot{q}_{1}^{-T} B^{-1}(q) \dot{q}_{1}^{-}$

We now state an important result related to impulsive braking.

Lemma 2 Consider the scalar function

$V=\frac{1}{2}\left[q_{1}^{T} K_{p} q_{1}+\dot{q}_{1}^{T} K_{d} \dot{q}_{1}+K_{e}\left(E-E_{\mathrm{des}}\right)^{2}\right]$

where $K_{p}$ and $K_{d}$ are diagonal positive definite constant matrices and $K_{e}$ is a positive constant. Impulsive braking results in a discontinuous jump in the function given by

$$
\begin{aligned}
\Delta V \triangleq\left(V^{+}-V^{-}\right) & \\
=-\frac{1}{2} \dot{q}_{1}^{-T} & {\left[\frac{1}{4}\left\{K_{e} \dot{q}_{1}^{-{ }^{T}} B^{-1}(q) \dot{q}_{1}^{-}\right\} B^{-1}(q)\right.} \\
& \left.+K_{d}+K_{e}\left(E^{+}-E_{\mathrm{des}}\right) B^{-1}(q)\right] \dot{q}_{1}^{-}
\end{aligned}
$$

where $V^{-}$and $V^{+}$are values of the function immediately before and after impulsive braking. Furthermore, if $\left[K_{d}+K_{e}\left(E^{+}-E_{\mathrm{des}}\right) B^{-1}(q)\right]$ is positive definite, then $\Delta V \leq 0$, and $\Delta V=0$ if and only if $\dot{q}_{1}^{-}=0$.

Proof: See section 8.2 of Appendix. 


\subsection{Impulsive Dynamical Model}

To stabilize the orbit in (1), we propose a control strategy comprised of continuous and impulsive inputs. The impulsive inputs will be used for impulsive braking of the active coordinates, i.e., $\dot{q}_{1}^{+}=0$. As a result, the change in the velocities can be obtained using (12) as follows:

$\Delta \dot{q}_{1}=0-\dot{q}_{1}^{-}=-\dot{q}_{1}^{-}$

$\Delta \dot{q}_{2}=\dot{q}_{2}^{+}-\dot{q}_{2}^{-}=\left(1 / M_{22}\right) M_{12}^{T} \dot{q}_{1}^{-}$

In addition to the impulsive braking inputs, we will reset the passive coordinate $q_{2}$ periodically to confine it to the compact set $[-3 \pi / 2, \pi / 2] 2$. To describe the dynamics of our system, we adopt the state-dependent impulsive dynamical model in [16, pg.20]:

$$
\begin{aligned}
\dot{x}(t) & =f_{c}[x(t)], \quad x(0)=x_{0}, & & x(t) \notin \mathcal{Z} \\
\Delta x(t) & =f_{d}[x(t)], & & x(t) \in \mathcal{Z}
\end{aligned}
$$

where $\mathcal{Z}$ defines the set where the impulsive inputs are applied and/or periodic resetting occurs. For our system,

$$
\begin{aligned}
x(t) & \triangleq\left[\begin{array}{llll}
q_{1}^{T} & q_{2} & \dot{q}_{1}^{T} & \dot{q}_{2}
\end{array}\right]^{T} \in \mathcal{D} \subseteq R^{2 n} \\
\Delta x(t) & \triangleq x\left(t^{+}\right)-x\left(t^{-}\right)
\end{aligned}
$$

In the above expression, $\mathcal{D}$ is the open set where $q_{2} \in$ $(a, b), a<-3 \pi / 2, b>\pi / 2$, and $x\left(t^{-}\right), x\left(t^{+}\right)$are the values of the state variables immediately before and after application of impulsive inputs or coordinate resetting. Using (9), (12) and (17), it can be shown

$$
\begin{aligned}
& f_{c}=\left[\begin{array}{c}
\dot{q}_{1} \\
\dot{q}_{2} \\
A(q, \dot{q})+B(q) u \\
-\left(1 / M_{22}\right)\left[M_{12}^{T}\{A(q, \dot{q})+B(q) u\}+h_{2}\right]
\end{array}\right]
\end{aligned}
$$

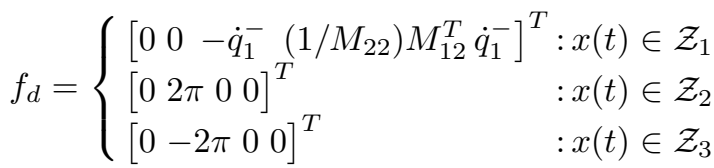

$\mathcal{Z}=\mathcal{Z}_{1} \cup \mathcal{Z}_{2} \cup \mathcal{Z}_{3}, \mathcal{Z}_{1}$ is the set where impulsive braking inputs are applied (to be defined in Theorem 2 where the control design will be presented), and $\mathcal{Z}_{2} \triangleq\left\{q_{2}=\right.$ $\left.-3 \pi / 2, \dot{q}_{2}<0\right\}$ and $\mathcal{Z}_{3} \triangleq\left\{q_{2}=\pi / 2, \dot{q}_{2}>0\right\}$ are the sets where coordinate resetting occurs.

We assume existence and uniqueness of the solution of (18) to exclude the possibility of complex phenomena such as Zeno switching [16,21. Simulations and experimental results presented later will validate this assumption.

\footnotetext{
2 This choice of the compact set is not unique.
}

\section{Control Design}

\subsection{Main Result}

For the objective in (1), we propose a control design comprised of continuous and intermittent impulsive braking inputs 3 . Theorem 2 provides the design of the continuous controller and defines the set $\mathcal{Z}_{1}$, where impulsive braking is applied. The proof of Theorem 2 is based on a Lyapunov-like function. The continuous controller is invoked as long as the derivative of the Lyapunovlike function is negative semi-definite; when this condition is not satisfied, impulsive braking is applied to produce negative jumps in the Lyapunov-like function. Before stating Theorem 2, we present an invariant set theorem [16, pg.38] that will be used in the proof of Theorem 2 and state one Assumption.

Theorem 1 [16, pg.38] Consider the impulsive dynamical system given by (18), assume that $\mathcal{D}_{c} \subset \mathcal{D}$ is a compact positively invariant set with respect (18), and assume that there exists a continuously differentiable function $W: \mathcal{D} \rightarrow R$ such that

$[\partial W(x) / \partial x] f_{c}(x) \leq 0, \quad x \in \mathcal{D}_{c}, x \notin \mathcal{Z}$

$W\left(x+f_{d}(x)\right) \quad \leq W(x), x \in \mathcal{D}_{c}, x \in \mathcal{Z}$

Let $\mathcal{R} \triangleq\left\{x \in \mathcal{D}_{c}: x \notin \mathcal{Z},[\partial W(x) / \partial x] f_{c}(x)=0\right\} \cup$ $\left\{x \in \mathcal{D}_{c}: x \in \mathcal{Z}, W\left(x+f_{d}(x)\right)=W(x)\right\}$ and let $\mathcal{M}$ denote the largest invariant set contained in $\mathcal{R}$. If $x_{0} \in$ $\mathcal{D}_{c}$, then $x(t) \rightarrow \mathcal{M}$ as $t \rightarrow \infty$.

Assumption 3 For the system in (7) subjected to continuous control, $q_{2}$ is constant if $\dot{q}_{1}$ and $\dot{u}$ are identically zero.

Remark 4 Assumption 3 implies that the active and passive generalized coordinates are dynamically coupled. Due to this coupling, the active generalized coordinates cannot be held stationary by constant generalized forces when the passive generalized coordinate is non-stationary. The existence of such coupling has been verified for an inverted pendulum on a cart [27, rotary pendulum 34, pendubot, acrobot, and reaction-wheel pendulum; in this paper it is shown for the tiptoebot.

Theorem 2 For the impulsive dynamical system defined by (18), (19) and (20), and $x_{0} \in \mathcal{D}$ such that

$-3 \pi / 2<q_{2}(0)<\pi / 2$

3 It is assumed that the active DOFs will have a friction brake such that they can be stopped instantaneously. 
the following choice of control design:

$$
\begin{gathered}
u=-\left[\left(K_{d}+K_{c}\right) B(q)+K_{e}\left(E-E_{\mathrm{des}}\right) I\right]^{-1} \times \\
{\left[K_{p} q_{1}+\left(K_{d}+K_{c}\right) A(q, \dot{q})\right]} \\
\mathcal{Z}_{1}=\left\{x(t) \mid[A(q, \dot{q})+B(q) u]^{T} K_{c} \dot{q}_{1} \leq 0, \dot{q}_{1} \neq 0\right\}
\end{gathered}
$$

where $I$ is the identity matrix and $K_{c}$ is a diagonal positive-definite matrix, guarantees asymptotic stability of the orbit in (1) if the gain matrices $K_{p}, K_{d}$ and $K_{e}$ satisfy the following conditions:

(i) $\left[K_{d}+K_{e}\left(E-E_{\mathrm{des}}\right) B^{-1}(q)\right]$ is positive definite for all $q$ and $\dot{q}$,

(ii) If $q_{1}^{*}$ and $q_{2}^{*}$ are constant values of $q_{1}$ and $q_{2}$, then the following system of equations:

$$
\begin{aligned}
& {\left[\frac{\partial \mathcal{F}}{\partial q_{1}}\right]_{q=q^{*}}^{T}=-\frac{K_{p} q_{1}^{*}}{K_{e}\left[\mathcal{F}\left(q^{*}\right)-E_{\mathrm{des}}\right]}} \\
& {\left[\frac{\partial \mathcal{F}}{\partial q_{2}}\right]_{q=q^{*}}=0}
\end{aligned}
$$

yields a finite number of solutions with $q_{1}^{*}=0$, and

(iii) For all possible solutions of $q_{2}^{*}$ obtained from (ii) and for the function $V$ in (15), the following inequality is satisfied

$V(t=0)<\min \left\{V \mid q_{1}=0, \dot{q}_{1}=0, E \in S_{E} \backslash\left\{E_{\mathrm{des}}\right\}\right\}$

where $S_{E}$ is the set of values of $E$ evaluated at $q_{1}=0, q_{2}=q_{2}^{*}, \dot{q}=0$.

Proof: Consider the Lyapunov-like function $V$ defined in (15); $V$ is zero on the orbit defined in (11) and positive everywhere else. The time derivative of $V$ is

$$
\begin{aligned}
\dot{V} & =q_{1}^{T} K_{p} \dot{q}_{1}+\ddot{q}_{1}^{T} K_{d} \dot{q}_{1}+K_{e}\left(E-E_{\mathrm{des}}\right) \dot{E} \\
& =\left[q_{1}^{T} K_{p}+\ddot{q}_{1}^{T} K_{d}+K_{e}\left(E-E_{\mathrm{des}}\right) u^{T}\right] \dot{q}_{1}
\end{aligned}
$$

where $\dot{E}=u^{T} \dot{q}_{1}$ follows from the passivity property of underactuated Euler-Lagrange systems - see 32] and proposition 2.5 of 35 . By substituting $\ddot{q}_{1}$ from (9a) in (24) and using the symmetry of $B(q)$, we get

$$
\begin{aligned}
\dot{V}=[ & q_{1}^{T} K_{p}+A^{T} K_{d} \\
& \left.+u^{T} B\left\{K_{d}+K_{e}\left(E-E_{\mathrm{des}}\right) B^{-1}\right\}\right] \dot{q}_{1}
\end{aligned}
$$

The following choice of $u$

$$
\begin{aligned}
u^{T}=-\left[q_{1}^{T} K_{p}+A^{T} K_{d}+\ddot{q}_{1}^{T} K_{c}\right] \times & \\
& {\left[B\left\{K_{d}+K_{e}\left(E-E_{\mathrm{des}}\right) B^{-1}\right\}\right]^{-1}, }
\end{aligned}
$$

\footnotetext{
4 The proof of the passivity property follows from the fact that the matrix $[\dot{M}-2 C]$ is skew-symmetric for our choice of generalized coordinates.
}

which is well defined based on condition $(i)$, results in

$\dot{V}=-\ddot{q}_{1}^{T} K_{c} \dot{q}_{1}$

Substitution of (9a) in (26) followed by algebraic manipulation gives the expression for $u$ in (23a). Substitution of (9a) in (27) gives

$\dot{V}=-[A(q, \dot{q})+B(q) u]^{T} K_{c} \dot{q}_{1}$

Based on the expression of $\dot{V}$, three cases may arise:

case (a): if $[A+B u]^{T} K_{c} \dot{q}_{1}>0$, then $\dot{V}<0$,

case (b): if $[A+B u]^{T} K_{c} \dot{q}_{1} \leq 0, \dot{q}_{1} \neq 0$, then $x \in \mathcal{Z}_{1}$ and impulsive braking is applied - see (23b). Since condition (i) is satisfied, Lemma 2 indicates that $V$ undergoes a discontinuous change $\Delta V$, where $\Delta V<0$, and

case (c): if $\dot{q}_{1}=0$, then $\dot{V}=0$.

For case (b), impulsive braking results in $\dot{q}_{1}=0$ at $t^{+}$ and the trajectories of the system leave $\mathcal{Z}_{1}$. If $\dot{q}_{1} \equiv 0$ for all $t>t^{+}$, the trajectories of the system remain outside $\mathcal{Z}_{1}$ and $\dot{V} \equiv 0$. If $\dot{q}_{1} \not \equiv 0$ for $t>t^{+}, V$ decreases since (27) implies

$$
\begin{gathered}
\dot{V}\left(t^{+}\right)=0, \quad \ddot{V}\left(t^{+}\right)=-\ddot{q}_{1}^{T}\left(t^{+}\right) K_{c} \ddot{q}_{1}\left(t^{+}\right)<0 \\
\Rightarrow \dot{V}(\tau)<0, \tau \in\left(t^{+}, t^{+}+\varepsilon\right)
\end{gathered}
$$

for some $\varepsilon>0$ since $K_{c}$ is positive-definite and $\ddot{q}_{1} \neq 0$. Case (c) implies that either $\dot{q}_{1} \equiv 0 \Rightarrow \dot{V} \equiv 0$, or $\dot{q}_{1} \not \equiv 0$ and $V$ continues to decrease again; this follows from our discussion of the nature of trajectories after impulsive braking. Cases (a), (b) and (c) imply that for $t>0$, $V(t) \leq V(0) \triangleq c$ and therefore the set

$\mathcal{D}_{c} \triangleq\{V \leq c\} \cap\left\{-3 \pi / 2 \leq q_{2} \leq \pi / 2\right\}$

is positively invariant.

Cases (a), (b) and (c) together satisfy the conditions in Theorem 1 with $\mathcal{D}_{c}$ defined above and $W(x)=V(x)$. Since (b) implies $\Delta V<0,\left\{x \in \mathcal{D}_{c}: x \in \mathcal{Z}, \Delta V=0\right\}$ is an empty set. Therefore, $x(t) \rightarrow \mathcal{M} \subset \mathcal{R}=\left\{x \in \mathcal{D}_{c}\right.$ : $x \notin \mathcal{Z}, \dot{V}=0\}$ as $t \rightarrow \infty$. From case (c), $\dot{V}=0$ implies $\dot{q}_{1}=0$ and thus $\mathcal{R}=\left\{x \in \mathcal{D}_{c}: \dot{q}_{1} \equiv 0\right\}$. In $\mathcal{R}, \ddot{q}_{1}=0$. Substitution of $\ddot{q}_{1}=0$ in (9a) and (26) yields

$$
\begin{aligned}
u^{T} & =-A^{T} B^{-1} \\
u^{T} B K_{d} & =-K_{e}\left(E-E_{\mathrm{des}}\right) u^{T}-q_{1}^{T} K_{p}-A^{T} K_{d}
\end{aligned}
$$

Substitution of (29a) into (29b) gives

$u^{T} K_{e}\left(E-E_{\mathrm{des}}\right)+q_{1}^{T} K_{p}=0$

The definition of $\mathcal{R}$ in Theorem 1 implies $V$ is constant in $\mathcal{R}$. Also, $q_{1}$ is constant and $\dot{q}_{1}=0$ in $\mathcal{R}$. Therefore, from the definition of $V$ in (15), we can claim that $E$ is constant in $\mathcal{R}$. Let $q_{1}^{*}$ and $E^{*}$ be the constant values of $q_{1}$ and $E$. We now discuss two cases that can arise: 
case 1: If $E^{*}=E_{\mathrm{des}}$, we have $q_{1}^{*}=0$ from (30). This implies that $\mathcal{M}$ is the orbit in (11).

case 2 : if $E^{*} \neq E_{\mathrm{des}}$, we get from (30)

$$
u \triangleq u^{*}=-\frac{K_{p} q_{1}^{*}}{K_{e}\left(E^{*}-E_{\mathrm{des}}\right)}
$$

where $u^{*}$ is the constant value of the continuous control in $\mathcal{R}$.

For case 2, both $q_{1}$ and $u$ are constants. Therefore, based on Assumption 3. we claim $q_{2}=q_{2}^{*}$ is a constant. It follows from (2) that $E^{*}=\mathcal{F}\left(q^{*}\right)$. Using (7) and (8), we can show that the trajectories in $\mathcal{R}$ satisfy

$$
\left[\frac{\partial \mathcal{F}}{\partial q_{1}}\right]_{q=q^{*}}^{T}=u^{*}, \quad\left[\frac{\partial \mathcal{F}}{\partial q_{2}}\right]_{q=q^{*}}=0
$$

Substituting the expression for $u^{*}$ from (31) in the above equation along with $E^{*}=\mathcal{F}\left(q^{*}\right)$, we can use condition (ii) to claim $q_{1}^{*}=0$. Using (15) and cases (a) and (b), we can claim that as $t \rightarrow \infty, V \rightarrow V^{*}$, where

$V^{*}=\frac{1}{2} K_{e}\left(E^{*}-E_{\mathrm{des}}\right)^{2} \leq V(t=0)$

where $E^{*} \in S_{E}$. Since $V^{*} \leq V(t=0)$, we can claim using condition (iii) that $E^{*}=E_{\mathrm{des}}$, i.e., $V^{*}=0$. Thus the largest invariant set $\mathcal{M}$ is the orbit defined in (1). This concludes the proof.

\subsection{Choice of Controller Gains}

It can be easily shown that condition (i) in Theorem 2 is satisfied if

$\left(1 / K_{e}\right) \lambda_{\min }\left(K_{d}\right)>\left[E_{\mathrm{des}}-\min (\mathcal{F})\right] \lambda_{\max }\left[B^{-1}(q)\right]$

where $\lambda_{\min }\left(K_{d}\right)$ and $\lambda_{\max }\left[B^{-1}(q)\right]$ are the minimum and maximum eigenvalues of $K_{d}$ and $\left[B^{-1}(q)\right.$ ]. Assumption 2 implies $\lambda_{\max }\left[B^{-1}(q)\right]$ and $\min (\mathcal{F})$ exist and therefore $K_{d}$ and $K_{e}$ can always be chosen to satisfy condition (i).

For the choice of $K_{e}$ satisfying condition (i), $K_{p}$ has to be chosen to satisfy condition (ii). Although we do not prove that condition (ii) can be simultaneously satisfied for the general case, several combinations of gains $\left(K_{p}, K_{d}, K_{e}\right)$ were found to exist for the inverted pendulum on a cart [27. The authors have independently verified that condition (ii) can be easily satisfied for several other underactuated mechanical systems, namely, the pendubot, the acrobot, and the reaction-wheel pendulum. It is shown in this paper that conditions (i) and (ii) can be simultaneously satisfied for the three-DOF Tiptoebot and the rotary pendulum. These examples indicate that condition (ii) is not restrictive.
Once the controller gains $K_{p}, K_{d}$ and $K_{e}$ have been chosen to satisfy conditions (i) and (ii) in Theorem 2 . condition (iii) imposes no additional restrictions on the gains but simply provides an estimate of the region of attraction of the orbit. Since $K_{c}$ does not appear in conditions (i)-(iii), it can be chosen without restriction.

\section{Illustrative Example - The Tiptoebot}

\subsection{System Description}

Consider the tiptoebot shown in Fig 1- it is a humanlike three DOF underactuated system with one passive joint. The three links are analogous to the lower leg, the upper leg, and the upper body comprised of the torso and head of a human. The knee joint connecting the upper and lower legs, and the hip joint connecting the upper body and upper leg are actuated; the torques applied by the actuators in these joints are assumed to be positive in the counter-clockwise direction and are denoted by $\tau_{2}$ and $\tau_{3}$. The toe provides a simple point of support and is modeled as a passive joint. The lower leg, upper leg, and upper body have link lengths $\ell_{1}, \ell_{2}$ and $\ell_{3}$ and masses $m_{1}, m_{2}$ and $m_{3}$ respectively. In this study, for the sake of simplicity, each link is assumed to be a rigid massless rod with a point mass attached to its distal end. The joint angles of the links are denoted by $\theta_{1}, \theta_{2}$ and $\theta_{3}$ and are assumed to be positive in the counter-clockwise direction; $\theta_{1}$ is measured relative to the $x$-axis whereas $\theta_{2}$ and $\theta_{3}$ are measured relative to the first and second links. The dynamics of the system is presented next.

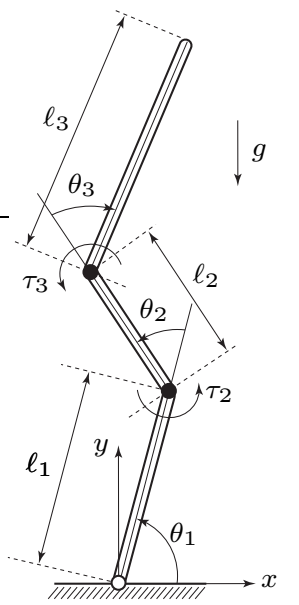

Fig. 1: The three-link underactuated tiptoebot has two active joints and one passive joint. 


\subsection{Tiptoebot Dynamics and Control Objective}

Using the following definition for the joint angles

$q_{1}^{T}=\left[\theta_{2} \theta_{3}\right]^{T}, \quad q_{2}=\theta_{1}$

the dynamics of the tiptoebot can be expressed in the form of (7); the components of mass matrix in (3) are

$$
\begin{aligned}
M_{11} & =\left[\begin{array}{cc}
\alpha_{2}+\alpha_{3}+2 \alpha_{5} \cos \theta_{3} & \alpha_{3}+\alpha_{5} \cos \theta_{3} \\
\alpha_{3}+\alpha_{5} \cos \theta_{3} & \alpha_{3}
\end{array}\right] \\
M_{12} & =\left[\begin{array}{c}
\alpha_{2}+\alpha_{3}+\alpha_{4} \cos \theta_{2}+2 \alpha_{5} \cos \theta_{3}+\alpha_{6} \cos \left(\theta_{2}+\theta_{3}\right) \\
\alpha_{3}+\alpha_{5} \cos \theta_{3}+\alpha_{6} \cos \left(\theta_{2}+\theta_{3}\right)
\end{array}\right] \\
M_{22} & =\alpha_{1}+\alpha_{2}+\alpha_{3} \\
& +2\left[\alpha_{4} \cos \theta_{2}+\alpha_{5} \cos \theta_{3}+\alpha_{6} \cos \left(\theta_{2}+\theta_{3}\right)\right]
\end{aligned}
$$

where $\alpha_{i}, i=1,2, \cdots, 6$, are lumped parameters, defined as follows:

$$
\begin{array}{ll}
\alpha_{1} \triangleq m_{1}\left(\ell_{1}^{2}+\ell_{2}^{2}+\ell_{3}^{2}\right), & \alpha_{2} \triangleq\left(m_{2}+m_{3}\right) \ell_{2}^{2} \\
\alpha_{3} \triangleq m_{3} \ell_{3}^{2}, & \alpha_{4} \triangleq m_{2} \ell_{1} \ell_{2}+m_{3} \ell_{1} \ell_{2} \\
\alpha_{5} \triangleq m_{3} \ell_{2} \ell_{3}, & \alpha_{6} \triangleq m_{3} \ell_{1} \ell_{3}
\end{array}
$$

The sum of Coriolis, centrifugal and gravitational force terms, $h_{1}$ and $h_{2}$, can be obtained using (8), where $\mathcal{F}(q)$ has the expression

$$
\begin{aligned}
\mathcal{F}= & \beta_{1} \sin \theta_{1}+\beta_{2} \sin \left(\theta_{1}+\theta_{2}\right)+\beta_{3} \sin \left(\theta_{1}+\theta_{2}+\theta_{3}\right) \\
& \beta_{1} \triangleq\left(m_{1}+m_{2}+m_{3}\right) \ell_{1} g \\
& \beta_{2} \triangleq\left(m_{2}+m_{3}\right) \ell_{2} g, \quad \beta_{3} \triangleq m_{3} \ell_{3} g
\end{aligned}
$$

The control input is defined as $u=\left[\begin{array}{ll}\tau_{2} & \tau_{3}\end{array}\right]^{T}$. In the compact set $\theta_{1} \in[-3 \pi / 2, \pi / 2]$, as defined in section 3.3 , the upright equilibrium configuration of the tiptoebot is defined by

$$
\theta_{1}=-3 \pi / 2 \text { or } \pi / 2,
$$

$\left[\theta_{2} \theta_{3} \dot{\theta}_{1} \dot{\theta}_{2} \dot{\theta}_{3}\right]=\left[\begin{array}{lllll}0 & 0 & 0 & 0 & 0\end{array}\right]$

is unstable, but can be stabilized, by a linear controller, for example. The stabilized equilibrium will typically have a finite region of attraction; therefore, to stabilize from an arbitrary initial configurations, we first use the controller in section 3 to stabilize an energy level set that intersects the region of attraction. The obvious choice for such a level set is the one where $E_{\text {des }}$ equals the potential energy of the system at the equilibrium. Substitution of $\theta_{1}=-3 \pi / 2$ or $\pi / 2$ and $\theta_{2}=\theta_{3}=0$ in (35) yields $E_{\mathrm{des}}=\beta_{1}+\beta_{2}+\beta_{3}$. The control objective in (1) can therefore be written as

$\theta_{2}=\theta_{3}=0, \dot{\theta}_{2}=\dot{\theta}_{3}=0, E_{\mathrm{des}}=\left(\beta_{1}+\beta_{2}+\beta_{3}\right)$

The feasibility of our control design is discussed next.

\subsection{Selection of Controller Gains}

The initial configuration of the tiptoebot is taken as

$$
\left[\theta_{1} \theta_{2} \theta_{3} \dot{\theta}_{1} \dot{\theta}_{2} \dot{\theta}_{3}\right]=[0 \pi \pi 000]
$$

In this configuration, the tiptoebot is coiled up: the first link is horizontal, the second link folds back on the first link, and the third link folds back on the second link. The links were chosen to have the same mass $m_{1}=$ $m_{2}=m_{3}=0.1 \mathrm{~kg}$ and the same length $\ell_{1}=\ell_{2}=\ell_{3}=$ $0.6 \mathrm{~m}$. For this choice of mass and length parameters, the lumped parameters of the tiptoebot, defined in (34) and (35), are provided in Table 1 below:

Table 1: Tiptoebot lumped parameters in SI units

\begin{tabular}{|l|l|l|l|l|l|}
\hline$\alpha_{1}$ & 0.108 & $\alpha_{4}$ & 0.072 & $\beta_{1}$ & 1.764 \\
\hline$\alpha_{2}$ & 0.072 & $\alpha_{5}$ & 0.036 & $\beta_{2}$ & 1.176 \\
\hline$\alpha_{3}$ & 0.036 & $\alpha_{6}$ & 0.036 & $\beta_{3}$ & 0.588 \\
\hline
\end{tabular}

The passive joint of the tiptoebot is revolute and therefore assumption 1 hold good. From the expressions in (33) and (35), it can be verified that assumption 2 holds good. Assumption 3 also holds good - this is discussed in the Appendix 8.3 ,

The following choice of gains satisfy condition (i) and (ii):

$K_{p}=\left[\begin{array}{cc}70 & 0 \\ 0 & 70\end{array}\right], \quad K_{d}=\left[\begin{array}{cc}2.8 & 0 \\ 0 & 2.8\end{array}\right], \quad K_{e}=2.2$

Condition (ii) results in $\theta_{2}^{*}=\theta_{3}^{*}=0$, which upon substitution in (7b) and (8b) yields

$\frac{\partial \mathcal{F}}{\partial q_{2}}=0 \quad \Rightarrow \quad \cos \theta_{1}^{*}=0$

From section 3.3 we know that $q_{2}$ lies in the compact set $[-3 \pi / 2, \pi / 2]$. Thus $\theta_{1}$ lies in the same compact set - see (32). In this set, the possible solutions of (39) are $\theta_{1}^{*}=\{-3 \pi / 2,-\pi / 2, \pi / 2\}$. For $\theta_{1}^{*}=-3 \pi / 2$ or $\pi / 2$, and $\theta_{2}^{*}=\theta_{3}^{*}=0$, we know that $E=E_{\text {des }}$. Therefore, to satisfy condition (iii), we use $\theta_{1}^{*}=-\pi / 2$; this results in the following inequality

$$
\begin{aligned}
V(t=0) & <2 K_{e}\left[E\left(q_{1}^{*}=0, q_{2}^{*}=-\pi / 2\right)-E_{\mathrm{des}}\right]^{2} \\
& =2 K_{e}\left(\beta_{1}+\beta_{2}+\beta_{3}\right)^{2}
\end{aligned}
$$

For the initial configuration in (37), $K_{e}$ in (38) satisfies the inequality above. The matrix $K_{c}$ was chosen as

$$
K_{c}=\left[\begin{array}{cc}
1.2 & 0 \\
0 & 1.2
\end{array}\right]
$$




\subsection{Simulation Results}

For the initial configuration in (37) and controller gains in (38) and (40), the simulation results are shown in Figs 2 and 3. The effect of impulsive braking can be seen in Figs 2 (d) and (f), where $\dot{\theta}_{2}$ and $\dot{\theta}_{3}$ (the velocities of the active joints) jump to zero on multiple occasions. Each impulsive braking also results in a negative jump in the mechanical energy (follows from Lemma 1) which can be seen in Fig 2 (b). Since impulsive inputs cause no jumps in the joint angles, there is no change in $\theta_{1}$, $\theta_{2}$ and $\theta_{3}$ at the time of impulsive braking - see Figs 2 (a), (c) and (e). In Fig 2 (a), $\theta_{1}$ never leaves the set $[-3 \pi / 2, \pi / 2]$ and therefore virtual impulsive inputs are not applied.

While impulsive brakings cause negative jumps in the total energy $E$, the continuous-time controller in (23a) adds energy to the system; together, they converge the energy to the desired values $E_{\text {des }}$ - see Fig, 2 (b). The phase portrait of the passive joint is shown in Fig 3 (a). The jumps in the phase portrait (vertical drops in $\dot{\theta}_{1}$, twice) is due to impulsive braking. The variation of the Lyapunov-like function $V$ with time is shown in Fig 3 (b) - it can be seen that $V$ decreases monotonically due to the action of the continuous-time controller and undergoes negative jumps intermittently due to impulsive brakings. The continuous controller and impulsive brakings work together to converge $V$ to zero.

The gain matrices in (38) and (40) were chosen such that convergence to the desired level set is fast. The simulation results indicate that the system trajectories (a)

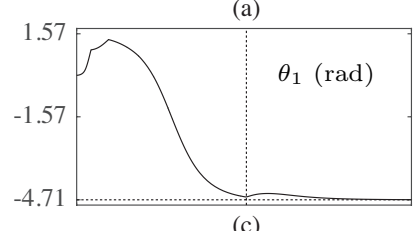

(c)

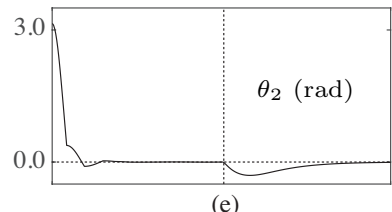

(e)

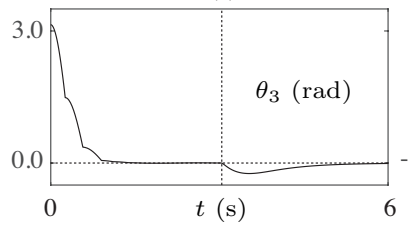

(b)

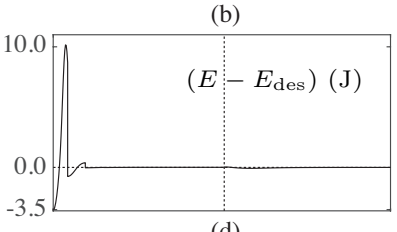

(d)
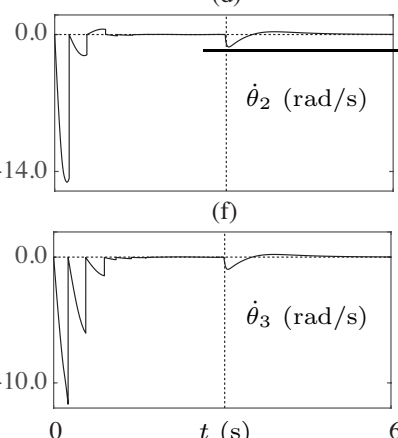

Fig. 2: Plots of the joint angles $\theta_{1}, \theta_{2}, \theta_{3}$, error in the desired energy $\left(E-E_{\mathrm{des}}\right)$, and the active joint velocities $\dot{\theta}_{2}, \dot{\theta}_{3}$ of the Tiptoebot. (a)

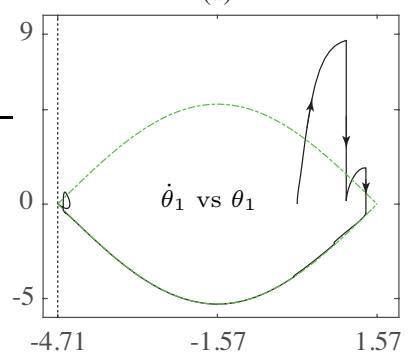

(b)

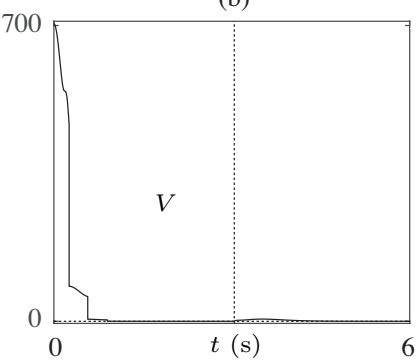

Fig. 3: Plots showing (a) phase portrait of passive joint angle $\theta_{1}$, and (b) variation of the Lyapunov-like function $V$. The desired level set is shown using dashed green line in (a).

reach a close neighborhood of the desired level set very quickly, at approximately $3 \mathrm{~s}$. For stabilization of the equilibrium in (37), a linear controller was designed using LQR. The matrices $Q$ and $R$ of the algebraic Ricatti equation were chosen to be $I_{6 \times 6}$ and $2 I_{2 \times 2}$, where $I_{k \times k}$ is the identity matrix of size $k$. The linear controller was invoked when the following conditions were simultaneously satisfied: $V \leq 0.05$ and $\left|\theta_{1}-\pi / 2\right| \leq 0.05$.

\section{Experimental Validation}

\subsection{System Description}

Experiments were done with a rotary pendulum. As shown in Fig 4. the system is comprised of a horizontal arm OA of mass $m_{a}$ and length $\ell_{a}$, which rotates about point $O$, and a pendulum of mass $m_{p}$ and length $\ell_{p}$, that rotates about point $\mathrm{A}$. The center-of-mass of the horizontal arm is located at a distance $d_{a}$ from $O$ and the center-of-mass of the pendulum is located at a distance $d_{p}$ from A. The horizontal arm is actively controlled by an external torque $\tau$ and its angular displacement about the $z$ axis is denoted by $\phi$. The pendulum is passive and its angular displacement about the $\varepsilon_{r}$ axis is

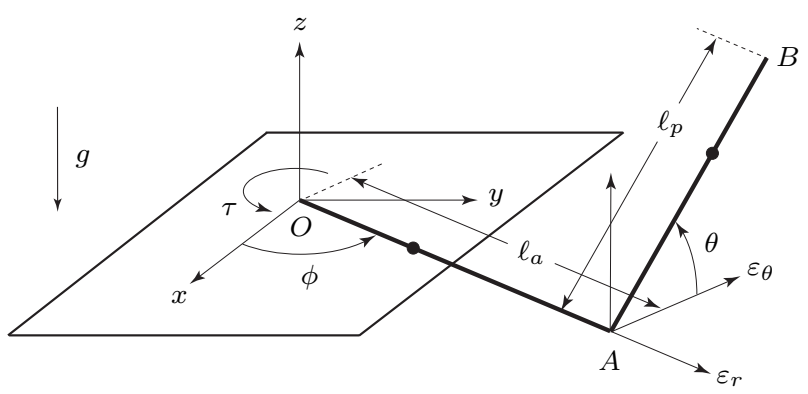

Fig. 4: Schematic of a rotary pendulum. 
denoted by $\theta$. The accleration due to gravity is denoted by $g$. With the following definition:

$\left.\left[\begin{array}{ll}q_{1} & q_{2}\end{array}\right]^{T}=[\phi \theta]\right]^{T}$

the dynamics of the system can be expressed in the from given by (7), where $u=\tau$, and

$$
\begin{aligned}
M_{11} & =\gamma_{1}+\gamma_{2} \cos ^{2} \theta, \quad M_{12}=\gamma_{3} \sin \theta, \quad M_{22}=\gamma_{2} \\
h_{1} & =\gamma_{3} \cos \theta \dot{\theta}^{2}-\dot{\phi} \dot{\theta} \gamma_{2} \sin 2 \theta \\
h_{2} & =\gamma_{2} \dot{\phi}^{2} \sin \theta \cos \theta+\gamma_{4} \cos \theta \\
\gamma_{1} & \triangleq m_{a} d_{a}^{2}+m_{p} \ell_{a}^{2}, \quad \gamma_{2} \triangleq m_{p} d_{p}^{2} \\
\gamma_{3} & \triangleq-m_{p} \ell_{a} d_{p}, \quad \gamma_{4} \triangleq m_{p} g d_{p}
\end{aligned}
$$

The physical parameters of the experimental setup are

$\gamma_{1}=0.0120, \gamma_{2}=0.0042, \gamma_{3}=-0.0038, \gamma_{4}=0.1190$

The control torque was applied by a 24-Volt permanent magnet brushed DC motor 5 . The motor is driven by a power amplifier 6 operating in current mode. The motor torque constant is $37.7 \mathrm{mNm} / \mathrm{A}$ and the amplifier gain is $4.4 \mathrm{~A} /$ volt. An electromagnetic friction brak 7 was integrated to the shaft of the DC motor. In the OFF state, the brake engages a friction pad to the shaft of the motor which prevents the shaft from turning; in the ON state, the brake is disengaged and the motor shaft rotates freely. For impulsive braking, the brake was kept engaged till the active velocity $\dot{\phi}$ reached a close neighborhood of zero. The brake was powered $\mathrm{ON} / \mathrm{OFF}$ by sending command voltage signals through an n-channel mosfet. The rotary pendulum was interfaced with a dSpace DS1104 board and the Matlab/Simulink environment was used for real-time data acquisition and control with a sampling rate of $1 \mathrm{Khz}$. The angular positions of the links were measured using incremental optical encoders; the angular velocities were obtained by differentiating and low-pass filtering the position signals.

5 The motor manufacturer is Faulhaber Drive Systems. The motor has a gearbox with a reduction ratio of $3.71: 1$.

6 The amplifier is a product of Advanced Motion Control.

7 The electromagnetic brake is manufactured by Anaheim Automation, model BRK-20H-480-024. The brake can withhold torques up to $3.4 \mathrm{Nm}$.

\subsection{Selection of Controller Gains}

The total energy of the system is obtained from (2) as follows

$$
\begin{aligned}
E & =\frac{1}{2}\left(\gamma_{1}+\gamma_{2} \cos ^{2} \theta\right) \dot{\phi}^{2}+\frac{1}{2} \gamma_{2} \dot{\theta}^{2}+\gamma_{3} \sin \theta \dot{\phi} \dot{\theta}+\mathcal{F} \\
\mathcal{F} & =\gamma_{4} \sin \theta
\end{aligned}
$$

For the control objective in (1), we choose $E_{\text {des }}$ to be equal to the energy associated with the homoclinic orbit that contains the upright equilibrium

$[\phi \theta \dot{\phi} \dot{\theta}]=[0 \pi / 200]$ or $[0-3 \pi / 200]$

Using (44), the energy associated with the homoclinic orbit can be written as

$E_{\mathrm{des}}=\gamma_{4}$

The passive joint of the rotary pendulum is revolute and thus assumption 1 holds good. From the expressions in (42) and (44), it can be verified that assumption 2 holds good. Similar to the Tiptoebot, we can show that assumption 3 holds good. From (44) we know that $\mathcal{F}$ is only a function of $\theta$ and therefore condition (ii) is trivially satisfied resulting in the solution $\phi^{*}=0$. In the compact set $[-3 \pi / 2, \pi / 2]$, the possible solutions of $\theta^{*}$ obtained from condition (ii) are $\theta^{*}=$ $\{-3 \pi / 2,-\pi / 2, \pi / 2\}$. At $\theta^{*}=\pi / 2$ or $\theta^{*}=-3 \pi / 2$ and $\phi^{*}=0, E=E_{\mathrm{des}}$. Using condition (iii), we therefore get $\theta^{*}=-\pi / 2$; this implies that $K_{e}$ should be chosen to satisfy

$V(t=0)<2 K_{e} \gamma_{4}^{2}$

At the lower equilibrium configuration where $\left[\begin{array}{lll}\phi & \dot{\phi} & \dot{\theta}\end{array}\right]=$ $\left[\begin{array}{llll}0 & -\pi / 2 & 0 & 0\end{array}\right]$, we have $V=2 K_{e} \gamma_{4}^{2}$. This violates the inequality in (46). This implies that our controller cannot swing-up the pendulum when the system is exactly at the lower equilibrium.Therefore, in experiments, a small external perturbation was provided such that the system is not at the lower equilibrium at the initial time. For the experimental results presented herein, the initial configuration of the system after the perturbation was measured as

$[\phi(0) \theta(0) \dot{\phi}(0) \dot{\theta}(0)]^{T}=[0.01-1.420 .050]^{T}$

For the initial conditions in (47) and physical parameter values in (43), the following gains satisfied conditions (i)-(iii):

$K_{p}=0.5, \quad K_{c}=0.08, \quad K_{d}=0.3, \quad K_{e}=100$

For the above set of gains, the experimental results are presented next. 


\subsection{Experimental Results}

The experimental results are shown in Fig 5, The controller for level set stabilization was active for the first $20 \mathrm{~s}$. At the end of this period, the system trajectories reached a close neighborhood of the upright equilibrium

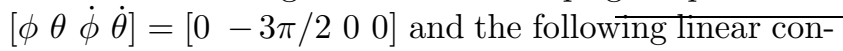
troller was invoked for stabilization:

$\tau_{s}=1.4 \phi-20.23(\theta+3 \pi / 2)+1.14 \dot{\phi}-1.98 \dot{\theta}$

The poles of the closed-loop system were located at $-37.0 \pm 20.0 i$ and $-1.0 \pm 1.2 i$.

The pulses shown on the top of Figs 5 (e) and (f) correspond to the time intervals when the brake was engaged (OFF) during level set stabilization. The brake was disengaged (ON) when the condition $|\dot{\phi}| \leq \mu$ was satisfied; the value of $\mu$ was chosen to be small, equal to $0.1 \mathrm{rad} / \mathrm{s}$. The time intervals required for braking were very short $(\approx 0.04 \mathrm{~s}$, on average); this implies that the brakings were impulsive in nature. The effect of impulsive braking can be seen in Fig 5 (b) where $\dot{\phi}$ jumps to almost zero value upon engagement of the brake on multiple occasions. It can be seen from Fig [5 (c) that the amplitude of the pendulum gradually increases and finally reaches a close neighborhood of the upright equilibrium configuration. The derivative of the Lyapunovlike function is shown in Fig 5 (f). It can be seen that (a)

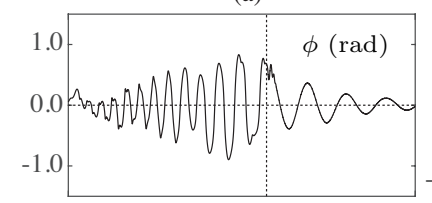

(c)

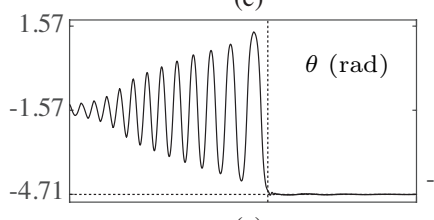

(e)

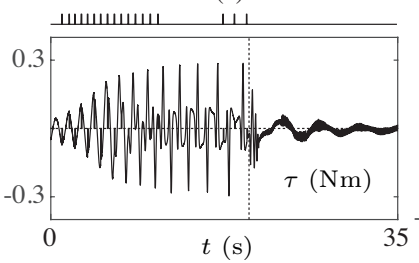

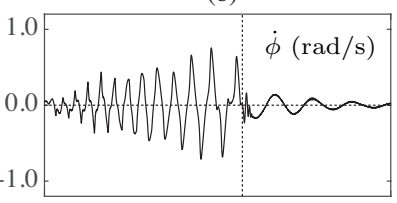

(d)

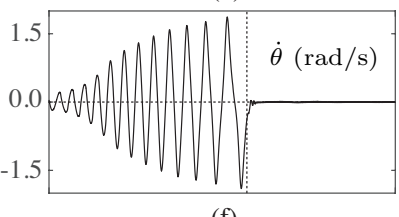

(f)

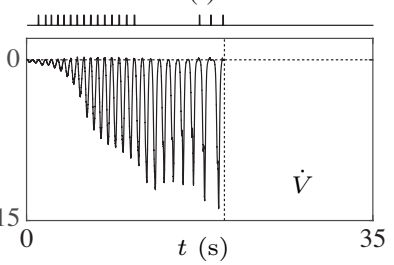

Fig. 5: Rotary pendulum experimental results: (a)-(d) are plots of joint angles and joint velocities, (e) control torque, and (f) derivative of Lyapunov-like function. The brake pulses are shown within plots (e) and (f), the peaks represent time intervals when the brakes were engaged.
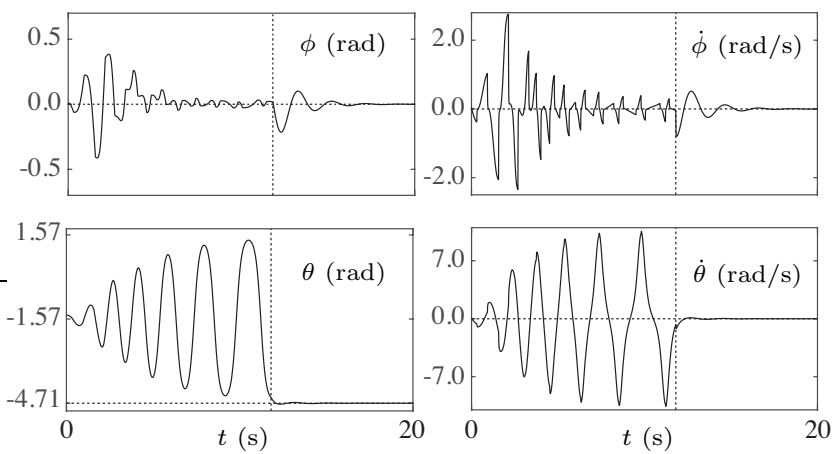

Fig. 6: Rotary pendulum simulation results.

$\dot{V}$ never becomes positive; this is because the brake is engaged every time when $\dot{V}$ is about to become positive 8 . Since $\dot{V}$ is always negative, $V$ decreases monotonically and stabilization of the level set is achieved. A plot of the motor torque is shown in Fig 5 (e). To minimize wear and tear of the brake, the commanded motor torque was set to zero when the brake was engaged. A video of this experiment has been uploaded as supplementary material.

Simulation results for the same set of initial conditions and controller gains in (47) and (48) are presented in Fig, 6. A comparison of Figs 5 and 6 indicate that the joint velocities in experiments are lower than those in simulations - this can be attributed to the presence of friction and other dissipative forces. The amplitude of the active joint $\phi$ is larger in experiments than simulations - this is due to the fact that the controller has to overcome the dissipative losses and additional energy is added through larger amplitude of motion. As expected, the time needed for stabilization is less in simulations than experiments.

Remark 5 For comparison, we considered the rotary pendulum example in [12. Taking identical initial conditions and physical parameters of the system therein, we simulated our controller with the gains

$K_{p}=0.20, \quad K_{d}=0.12, \quad K_{e}=50, \quad K_{c}=0.70$

The gains were tuned such that the magnitude of the motor torque did not exceed $0.3 \mathrm{Nm}$. The system trajectories converged to the desired level set in approx. 30 s. The controller in 12 took approx. $100 \mathrm{sec}$ and the magnitude of the maximum torque was $8 \mathrm{Nm}$. Our controller performed well, both in terms of motor torque requirement and speed of convergence. This better performance, however, comes at the cost of additional brake hardware.

8 When $|\dot{\phi}| \leq \mu \approx 0$, the brake is not engaged since $\dot{V} \approx 0$ see (27). 


\section{Conclusion}

A control strategy was presented for stabilization of energy level sets of underactuated systems with one passive DOF. The level set is defined with the help of a Lyapunov-like function that has been commonly used in the literature. Unlike existing energy-based methods, that have relied on continuous control inputs alone, our control strategy uses continuous control inputs and intermittent impulsive brakings. The continuous control is designed to make the time derivative of the Lyapunovlike function negative semi-definite. When this condition cannot be enforced, the impulsive inputs are invoked. This results in negative jumps in the Lyapunovlike function and guarantees its negative semi-definiteness under continuous control for some finite time interval. Thus, a combination of continuous and impulsive inputs guarantees monotonic convergence of the system trajectories to the desired energy level set, which can be periodic, or non-periodic as in the case of homoclinic orbits, depending on the choice of desired energy. More importantly, it allows us to develop a general framework for energy-based orbital stabilization, which is an important contribution of this paper. A set of conditions, that impose constraints on the choice of controller gains, have to be satisfied for applicability of the control strategy. These conditions are easily satisfied by systems commonly studied in the literature such as the pendubot, acrobot, inertia-wheel pendulum, and pendulum on a cart. In this paper, the control strategy was demonstrated in a three-DOF underactuated system using simulations and the two-DOF rotary pendulum using experiments. In experiments, impulsive brakings were not applied by the motor; instead, they were applied by a friction brake mounted co-axially with the motor shaft. This requires additional hardware but there are two important advantages of using the brake. In physical systems, impulsive inputs are implemented using high-gain feedback, which can result in actuator saturation. Since our impulsive control strategy requires the active velocities to be reduced to zero, a brake is a natural choice and it eliminates the possibility of motor torque saturation. The advantage of using a brake is also manifested in the time required for stabilization. A comparison of our approach with an approach in the literature shows significant reduction in the time for convergence for the same set of initial conditions. Our future work will focus on extension of our approach to orbital stabilization using virtual holonomic constraints.

\section{Appendix}

\subsection{Proof of Lemma 1}

The proof of Lemma 1 is provided here for the general case where the underactuated system has $m$ passive and $n-m$ active generalized coordinates, i.e. $q_{1} \in$ $R^{n-m}, q_{2} \in R^{m}$ and $u \in R^{n-m}$. The equation of motion has the form in (7) with $M_{11} \in R^{(n-m) \times(n-m)}$, $M_{22} \in R^{m \times m}, h_{1} \in R^{(n-m)}$, and $h_{2} \in R^{m}$. The change in the energy due to application of an impulsive input is equal to the change in the kinetic energy:

$$
\begin{aligned}
\Delta E & =\frac{1}{2} \dot{q}^{+{ }^{T}} M(q) \dot{q}^{+}-\frac{1}{2} \dot{q}^{-{ }^{T}} M(q) \dot{q}^{-} \\
& =\frac{1}{2}\left[\dot{q}_{1}^{{ }^{T}} M_{11} \dot{q}_{1}^{+}-\dot{q}_{1}^{-{ }^{T}} M_{11} \dot{q}_{1}^{-}\right] \\
& +\frac{1}{2}\left[\dot{q}_{2}^{{ }^{T}} M_{22} \dot{q}_{2}^{+}-\dot{q}_{2}^{-{ }^{T}} M_{22} \dot{q}_{2}^{-}\right] \\
& +\dot{q}_{1}^{+^{T}} M_{12} \dot{q}_{2}^{+}-\dot{q}_{1}^{-{ }^{T}} M_{12} \dot{q}_{2}^{-}
\end{aligned}
$$

The impulse manifold, given in (12) for $m \geq 1$, is

$\dot{q}_{2}^{+}=\dot{q}_{2}^{-}-M_{22}^{-1} M_{12}^{T}\left(\dot{q}_{1}^{+}-\dot{q}_{1}^{-}\right)$

Substitution of $\dot{q}_{2}^{+}$from A.2 into A.1 yields

$$
\begin{aligned}
\Delta E= & \frac{1}{2}\left[\dot{q}_{1}^{+{ }^{T}} M_{11} \dot{q}_{1}^{+}-\dot{q}_{1}^{-{ }^{T}} M_{11} \dot{q}_{1}^{-}\right] \\
+ & \frac{1}{2}\left[\dot{q}_{2}^{-}-M_{22}^{-1} M_{12}^{T}\left(\dot{q}_{1}^{+}-\dot{q}_{1}^{-}\right)\right]^{T} M_{22} \times \\
& {\left[\dot{q}_{2}^{-}-M_{22}^{-1} M_{12}^{T}\left(\dot{q}_{1}^{+}-\dot{q}_{1}^{-}\right)\right] } \\
- & \frac{1}{2} \dot{q}_{2}^{-{ }^{T}} M_{22} \dot{q}_{2}^{-}-\dot{q}_{1}^{{ }^{T}} M_{12} \dot{q}_{2}^{-} \\
+ & \dot{q}_{1}^{+{ }^{T}} M_{12}\left[\dot{q}_{2}^{-}-M_{22}^{-1} M_{12}^{T}\left(\dot{q}_{1}^{+}-\dot{q}_{1}^{-}\right)\right]
\end{aligned}
$$

Expanding, canceling, and regrouping the terms on the right-hand side of the above equation yields

$$
\begin{aligned}
\Delta E & =\frac{1}{2} \dot{q}_{1}^{+^{T}}\left[M_{11}-M_{12} M_{22}^{-1} M_{12}^{T}\right] \dot{q}_{1}^{+} \\
& -\frac{1}{2} \dot{q}_{1}^{-T}\left[M_{11}-M_{12} M_{22}^{-1} M_{12}^{T}\right] \dot{q}_{1}^{-}
\end{aligned}
$$

Similar to (10), $B(q)$ is defined for the general case as follows

$$
B(q)=\left[M_{11}-M_{12} M_{22}^{-1} M_{12}^{T}\right]^{-1}
$$

From the properties of the mass matrix $M(q)$, it can be shown that $B(q)$ is well-defined; also, it is symmetric and positive-definite, i.e., $B(q)=B^{T}(q)>0$. Substitution of (A.4) into A.3 gives (13). 


\subsection{Proof of Lemma 2}

Impulsive inputs result in no change in the generalized coordinates. Additionally, impulsive braking results in $\dot{q}_{1}^{+}=0$. Therefore, from the definition of $V$ in (15), $\Delta V$ for impulsive braking can be expressed as

$$
\begin{aligned}
\Delta V & =\frac{1}{2}\left[K_{e}\left(E^{+}-E_{\mathrm{des}}\right)^{2}-K_{e}\left(E^{-}-E_{\mathrm{des}}\right)^{2}\right. \\
\left.-\dot{q}_{1}^{-{ }^{T}} K_{d} \dot{q}_{1}^{-}\right] & \\
= & \frac{1}{2}\left[K_{e}\left(E^{+}+E^{-}-2 E_{\mathrm{des}}\right) \Delta E-\dot{q}_{1}^{-{ }^{T}} K_{d} \dot{q}_{1}^{-}\right] \\
= & \frac{1}{2}\left[K_{e}\left(2 E^{+}-\Delta E-2 E_{\mathrm{des}}\right) \Delta E-\dot{q}_{1}^{-T} K_{d} \dot{q}_{1}^{-}\right]
\end{aligned}
$$

where $\Delta E$ is defined in (13). Substitution of $\Delta E$ from (14) in the equation above yields

$$
\begin{aligned}
& \Delta V=-\frac{1}{2}\left[\left(\dot{q}_{1}^{-T} B^{-1} \dot{q}_{1}^{-}\right) K_{e}\left\{E^{+}-E_{\mathrm{des}}+\frac{1}{4} \dot{q}_{1}^{-{ }^{T}} B^{-1} \dot{q}_{1}^{-}\right\}\right. \\
& \left.+\dot{q}_{1}^{-T} K_{d} \dot{q}_{1}^{-}\right] \\
& =-\frac{1}{2} \dot{q}_{1}^{-{ }^{T}}\left[K_{e}\left\{E^{+}-E_{\mathrm{des}}+\frac{1}{4} \dot{q}_{1}^{-{ }^{T}} B^{-1} \dot{q}_{1}^{-}\right\} B^{-1}\right. \\
& \left.+K_{d}\right] \dot{q}_{1}^{-} \\
& =-\frac{1}{2} \dot{q}_{1}^{-T}\left[\frac{1}{4}\left\{K_{e} \dot{q}_{1}^{-T} B^{-1} \dot{q}_{1}^{-}\right\} B^{-1}\right. \\
& \left.+K_{d}+K_{e}\left(E^{+}-E_{\mathrm{des}}\right) B^{-1}\right] \dot{q}_{1}^{-}
\end{aligned}
$$

which is the same as in (16). Since $B$, defined in (A.4), is positive-definite, $\left\{K_{e} \dot{q}_{1}^{-{ }^{T}} B^{-1} \dot{q}_{1}^{-}\right\} B^{-1}$ is positive-definite. Therefore, if $\left[K_{d}+K_{e}\left(E^{+}-E_{\mathrm{des}}\right) B^{-1}(q)\right]$ is positivedefinite, $\Delta V \leq 0$ and $\Delta V=0$ iff $\dot{q}_{1}^{-}=0$.

\subsection{Assumption 3 holds for Tiptoebot}

A constant value of $u$ implies $\tau_{2}$ and $\tau_{3}$ are constants. A constant value of $q_{1}$ implies $\dot{\theta}_{2}=\dot{\theta}_{3}=0$ from (32). Substituting these conditions in (7), (8) and (2), we get

$$
\begin{aligned}
& M_{12} \ddot{q}_{2}+\dot{M}_{12} \dot{q}_{2}-\frac{1}{2}\left[\frac{\partial M_{22}}{\partial q_{1}}\right]^{T} \dot{q}_{2}^{2}+\left[\frac{\partial \mathcal{F}}{\partial q_{1}}\right]^{T}=u \\
& M_{22} \ddot{q}_{2}+\dot{M}_{22} \dot{q}_{2}-\frac{1}{2}\left[\frac{\partial M_{22}}{\partial q_{2}}\right] \dot{q}_{2}^{2}+\frac{\partial \mathcal{F}}{\partial q_{2}}=0 \\
& E=\frac{1}{2} M_{22} \dot{q}_{2}^{2}+\mathcal{F}
\end{aligned}
$$

From (33), it can be seen that $M_{12}$ and $M_{22}$ are only function of $q_{1}$, which is constant. Therefore, in A.5 $\dot{M}_{12}=\dot{M}_{22}=0$; also, $\left(\partial M_{22} / \partial q_{2}\right)=0$ since $M_{22}$ is not a function of $q_{1}$. Furthermore, from the passivity property of underactuated mechanical systems [32,35], we have $\dot{E}=u^{T} \dot{q}_{1}=0$. This implies $E$ is constant in (A.5). Manipulating A.5 to eliminate $\dot{q}_{2}$ and $\ddot{q}_{2}$, we get

$\left[\frac{\partial \mathcal{F}}{\partial q_{1}}\right]^{T}-\frac{M_{12}}{M_{22}} \frac{\partial \mathcal{F}}{\partial q_{2}}-\frac{(E-\mathcal{F})}{M_{22}}\left[\frac{\partial M_{22}}{\partial q_{1}}\right]^{T}=u$

From (33), it can be seen that $M_{12}$ and $M_{22}$ are functions of $q_{1}$ only; therefore, $M_{12}, M_{22}$, and $\left(\partial M_{22} / \partial q_{1}\right)^{T}$ are constants. Furthermore $u$ and $E$ are constants and $\mathcal{F}$ is a function of $q_{2}$ since $q_{1}$ is constant. Therefore (A.6) can be manipulated and written in the form

$\sin \left[q_{2}+c_{1}\right]=c_{2}$

where $c_{1}$ and $c_{2}$ are constants. This implies that $q_{2}$ is constant.

\section{Acknowledgements}

The authors gratefully acknowledge the support provided by the National Science Foundation, NSF Grant CMMI-1462118.

\section{Data Availability}

The datasets generated during and/or analysed during the current study are available from the corresponding author on reasonable request.

\section{Conflict of Interest}

The authors declare that they do not have any conflict of interest.

\section{References}

1. F. Plestan, J. W. Grizzle, E. R. Westervelt, and G. Abba, "Stable walking of a 7-dof biped robot," IEEE Transactions on Robotics and Automation, vol. 19, no. 4, pp. 653$668,2003$.

2. E. R. Westervelt, C. Chevallereau, J. H. Choi, B. Morris, and J. W. Grizzle, Feedback Control of Dynamic Bipedal Robot Locomotion. CRC press, 2007.

3. M. Maggiore and L. Consolini, "Virtual holonomic constraints for Euler- Lagrange systems," IEEE Transactions on Automatic Control, vol. 58, no. 4, pp. 1001-1008, 2013.

4. A. Shiriaev, J. W. Perram, and C. Canudas-de Wit, "Constructive tool for orbital stabilization of underactuated nonlinear systems: Virtual constraints approach," IEEE Transactions on Automatic Control, vol. 50, no. 8, pp. 1164-1176, 2005.

5. A. Mohammadi, M. Maggiore, and L. Consolini, "Dynamic virtual holonomic constraints for stabilization of closed orbits in underactuated mechanical systems," $A u$ tomatica, vol. 94, pp. 112-124, 2018. 
6. N. Kant and R. Mukherjee, "Orbital stabilization of underactuated systems using virtual holonomic constraints and impulse controlled Poincaré maps," Systems 83 Control Letters, vol. 146, p. 104813, 2020.

7. I. Fantoni, R. Lozano, and M. Spong, "Energy based control of the pendubot," IEEE Trans. on Automatic Control, vol. 45, no. 4, pp. 725-729, 2000.

8. X. Xin and T. Yamasaki, "Energy-based swing-up control for a remotely driven acrobot: Theoretical and experimental results," IEEE Transactions on Control Systems Technology, vol. 20, no. 4, pp. 1048-1056, 2012.

9. I. Fantoni, R. Lozano, and M. Spong, "Stabilization of the reaction wheel pendulum using an energy approach," in European Control Conference, 2001, pp. 2552-2557.

10. R. Lozano, I. Fantoni, and D. J. Block, "Stabilization of the inverted pendulum around its homoclinic orbit," Systems \& Control Letters, pp. 197-204, 2000.

11. H. Oka, Y. Maruki, H. Suemitsu, and T. Matsuo, "Nonlinear control for rotational movement of cart-pendulum system using homoclinic orbit," International Journal of Control, Automation and Systems, vol. 14, no. 5, pp. 12701279, 2016.

12. I. Fantoni and R. Lozano, "Stabilization of the Furuta pendulum around its homoclinic orbit," International Journal of Control, vol. 75, no. 6, pp. 390-398, 2002.

13. X. Xin and M. Kaneda, "Swing-up control for a 3-dof gymnastic robot with passive first joint: design and analysis," IEEE Transactions on Robotics, vol. 23, no. 6, pp. 1277-1285, 2007.

14. T. Albahkali, R. Mukherjee, and T. Das, "Swing-up control of the pendubot: An impulse-momentum approach," IEEE Transactions on Robotics, vol. 25, no. 4, pp. 975-982, 2009.

15. B. Brogliato, S. . Niculescu, and P. Orhant, "On the control of finite-dimensional mechanical systems with unilateral constraints," IEEE Transactions on Automatic Control, vol. 42, no. 2, pp. 200-215, Feb 1997.

16. W. M. Haddad, V. Chellaboina, and S. G. Nersesov, Impulsive and Hybrid Dynamical Systems. Princeton Series in Applied Mathematics, 2006.

17. R. I. Leine and N. Van de Wouw, Stability and Convergence of Mechanical Systems with Unilateral Constraints. Springer Science \& Business Media, 2007, vol. 36.

18. N. Van De Wouw and R. Leine, "Robust impulsive control of motion systems with uncertain friction," International Journal of Robust and Nonlinear Control, vol. 22, no. 4, pp. 369-397, 2012.

19. D. D. Bainov and P. S. Simeonov, Systems with Impulse Effect: Stability, Theory, and Applications. Ellis Horwood, 1989.

20. B. M. Miller and E. Y. Rubinovich, Impulsive Control in Continuous and Discrete-Continuous Systems. Springer Science \& Business Media, 2012.

21. R. Goebel, R. G. Sanfelice, and A. R. Teel, Hybrid Dynamical Systems: Modeling Stability, and Robustness. Princeton University Press, Princeton, NJ, 2012.
22. F. B. Mathis, R. Jafari, and R. Mukherjee, "Impulsive actuation in robot manipulators: Experimental verification of pendubot swing-up," IEEE Transactions on Mechatronics, vol. 19, no. 4, pp. 1469-1474, 2014.

23. R. Jafari, F. B. Mathis, R. Mukherjee, and H. Khalil, "Enlarging the region of attraction of equilibria of underactuated systems using impulsive inputs," IEEE Transactions on Control Systems Technology, vol. 24, no. 1, pp. 334-340, 2016.

24. N. Kant, R. Mukherjee, D. Chowdhury, and H. K. Khalil, "Estimation of the region of attraction of underactuated systems and its enlargement using impulsive inputs," IEEE Transactions on Robotics, vol. 35, no. 3, pp. 618632, June 2019.

25. N. Kant and R. Mukherjee, "Impulsive dynamics and control of the inertia-wheel pendulum," IEEE Robotics and Automation Letters, vol. 3, no. 4, pp. 3208-3215, 2018.

26. N. Kant, R. Mukherjee, and H. K. Khalil, "Swing-up of the inertia wheel pendulum using impulsive torques," in Proc. IEEE Conference on Decision and Control, 2017, pp. 5833-5838.

27. N. Kant, R. Mukherjee, and H. K. Khalil, "Stabilization of homoclinic orbits of two degree-of-freedom underactuated systems," in 2019 American Control Conference, July 2019, pp. 699-704.

28. N. Kant and R. Mukherjee, "Non-prehensile manipulation of a devil-stick: planar symmetric juggling using impulsive forces," Nonlinear Dynamics, vol. 103, no. 3, pp. 2409-2420, 2021.

29. Y. Li, C. Li, Z. He, and Z. Shen, "Estimating and enlarging the region of attraction of multi-equilibrium points system by state-dependent edge impulses," Nonlinear Dynamics, vol. 103, no. 3, pp. 2421-2436, 2021.

30. B. Brogliato, R. Lozano, B. Maschke, and O. Egeland, Dissipative Systems Analysis and Control. Springer, 2020.

31. L. L. Flynn, R. Jafari, and R. Mukherjee, "Active synthetic-wheel biped with torso," IEEE Transactions on Robotics, vol. 26, no. 5, pp. 816-826, 2010.

32. D. Liu, W. Guo, J. Yi, and D. Zhao, "Passivity-basedcontrol for a class of underactuated mechanical systems," in IEEE International Conference on Intelligent Mechatronics and Automation, 2004, pp. 50-54.

33. B. Brogliato, Nonsmooth Mechanics. Models, Dynamics and Control. Springer Nature Switzerland AG, 2020.

34. N. Kant, "Exploiting impulsive inputs for stabilization of underactuated robotic systems: Theory and experiments," Ph.D. dissertation, Michigan State University, 2020.

35. R. Ortega, J. A. L. Perez, P. J. Nicklasson, and H. J. SiraRamirez, Passivity-based control of Euler-Lagrange systems: Mechanical, Electrical and Electromechanical Applications. Springer Science \& Business Media, 2013. 
Figures

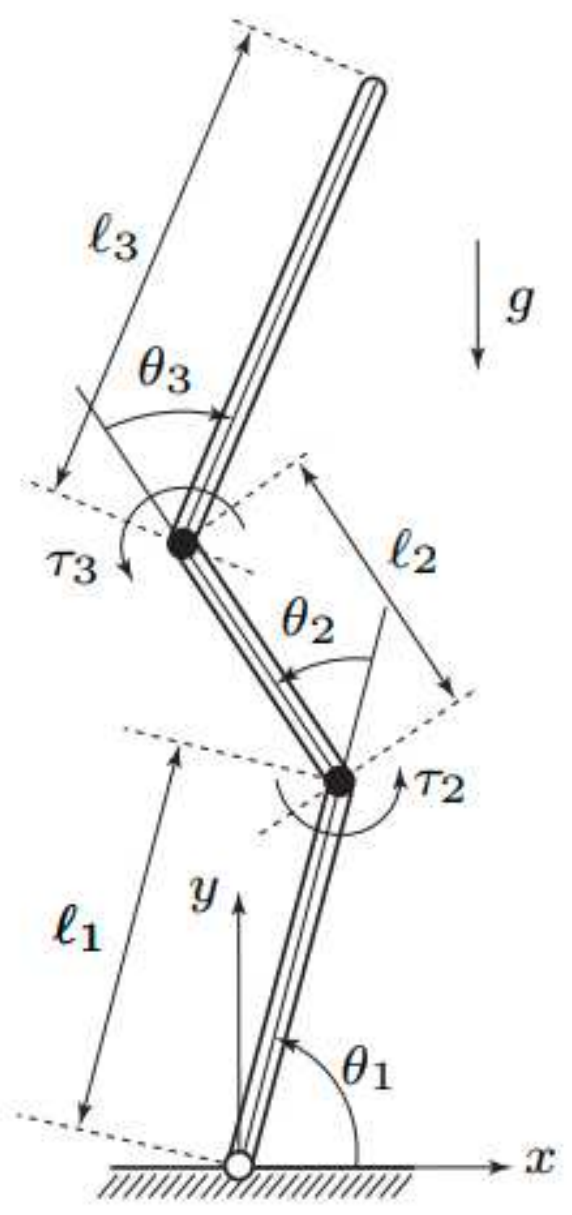

\section{Figure 1}

The three-link underactuated tiptoebot has two active joints and one passive joint. 
(a)

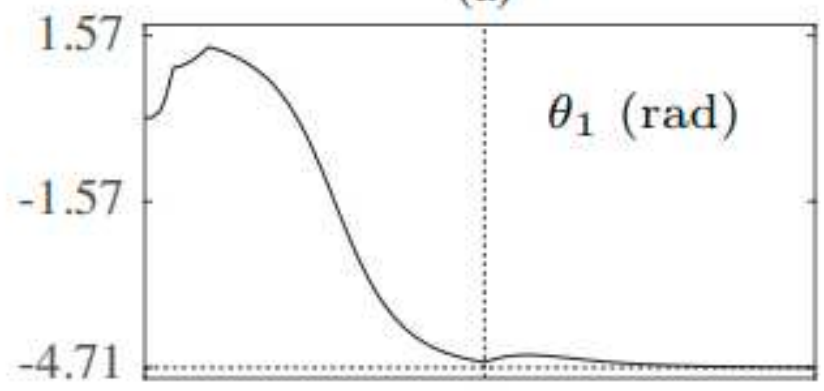

(c)

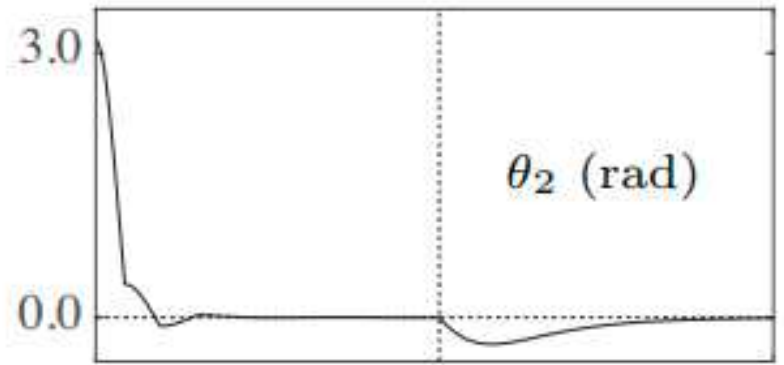

(e)

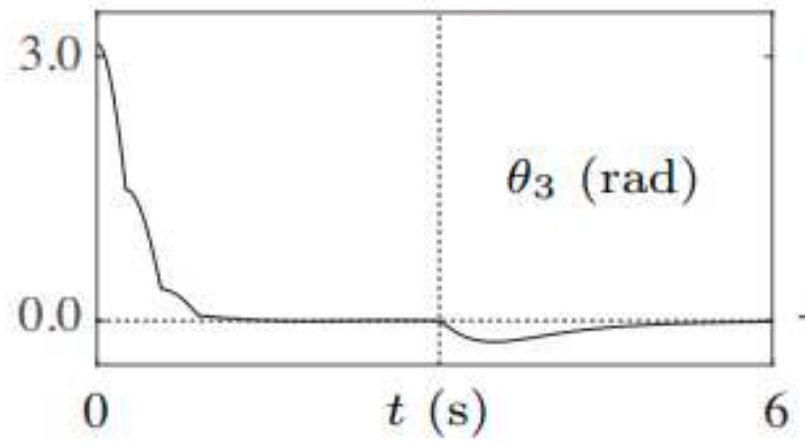

(b)

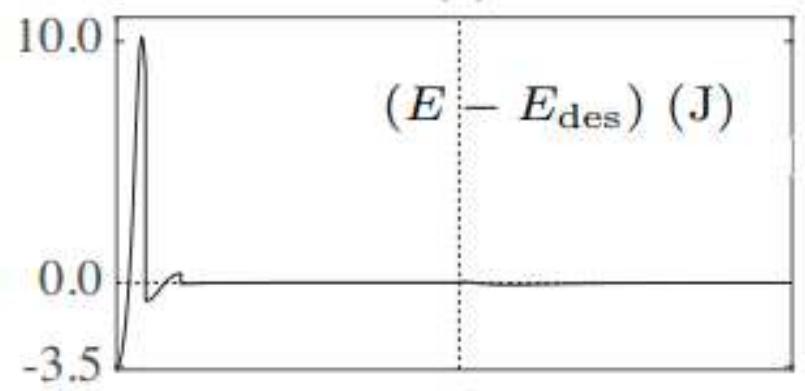

(d)

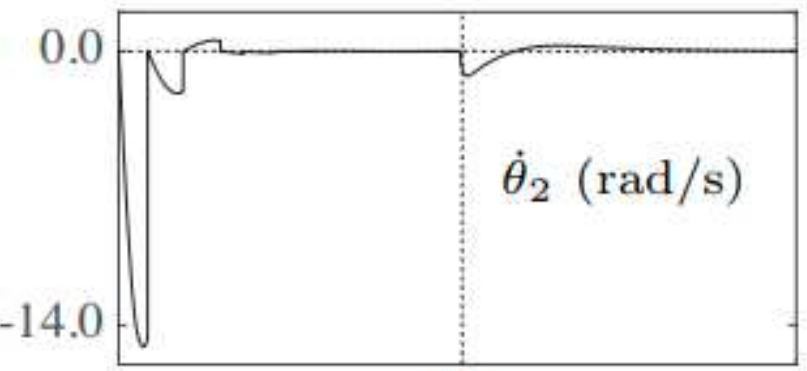

(f)

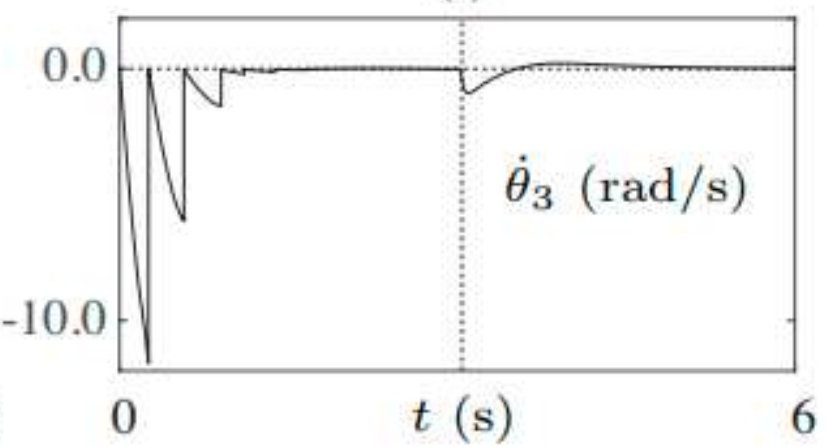

Figure 2

Please see the Manuscript PDF file for the complete figure caption 
(a)

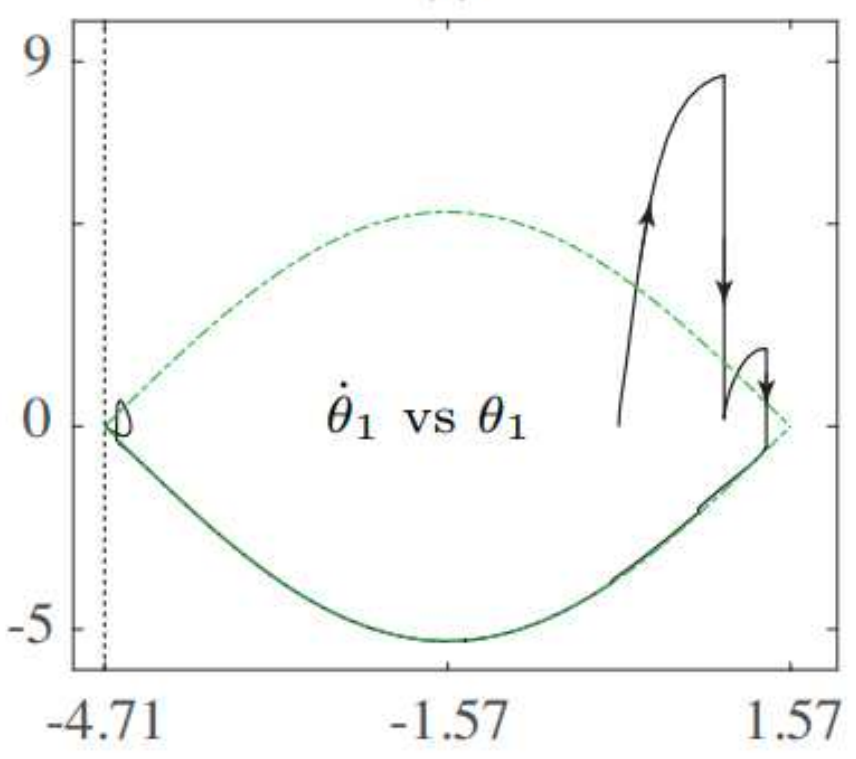

(b)

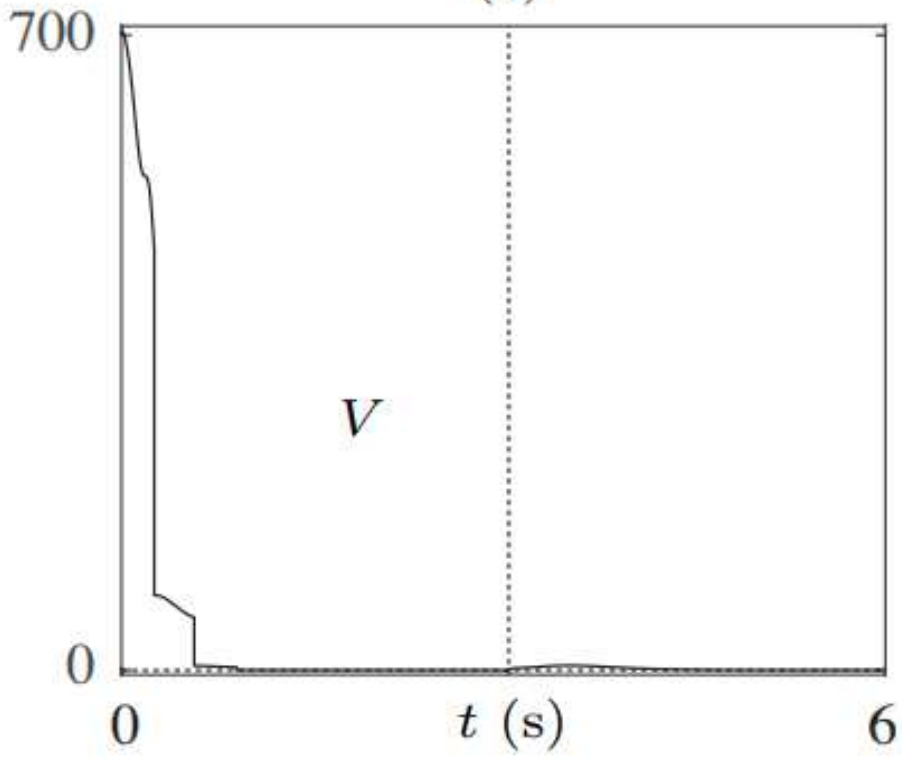

\section{Figure 3}

Please see the Manuscript PDF file for the complete figure caption

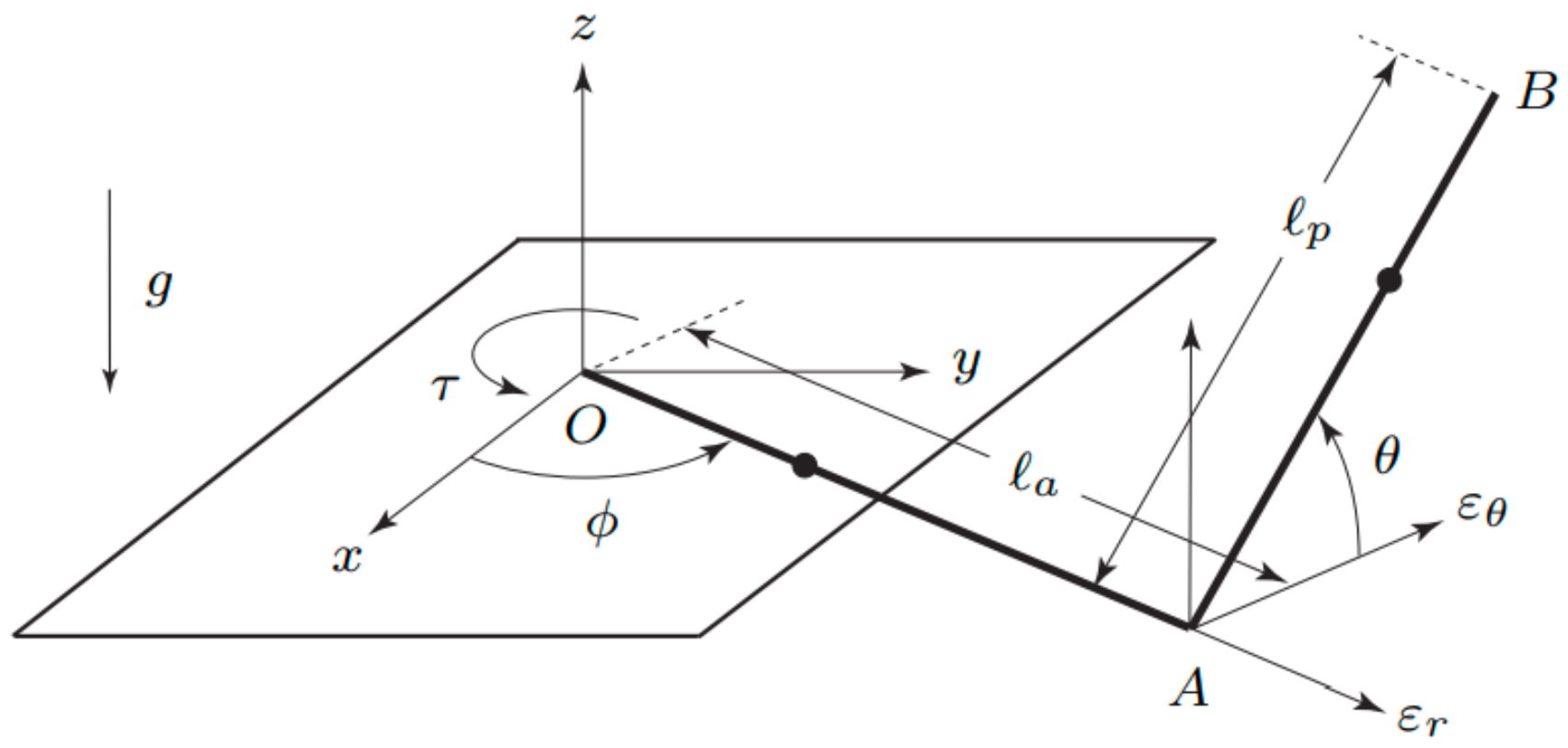

Figure 4

Schematic of a rotary pendulum. 
(a)

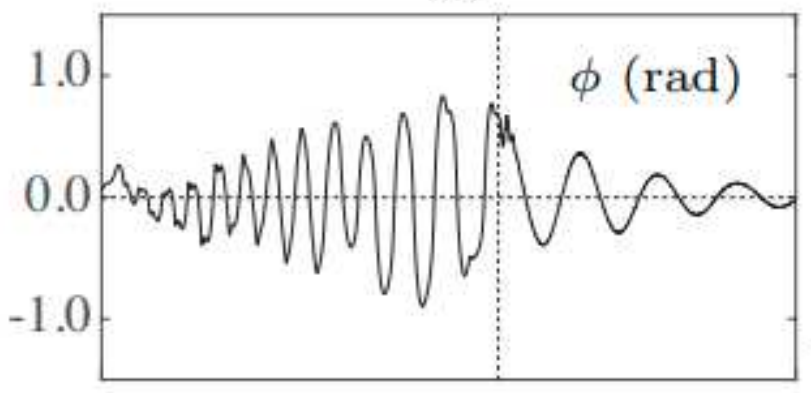

(c)

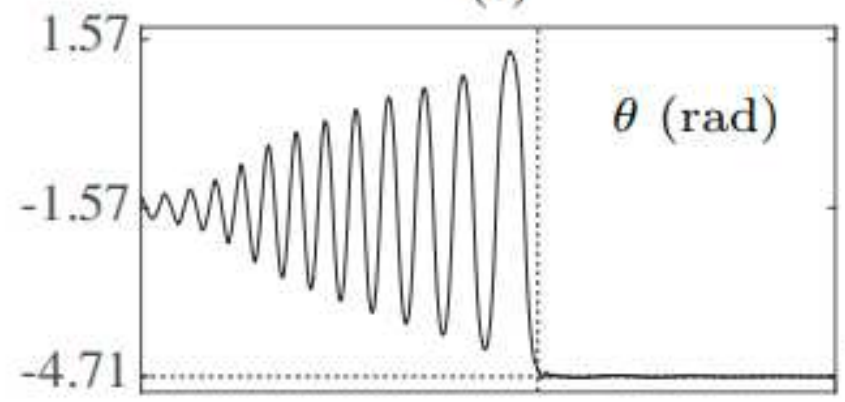

(e)

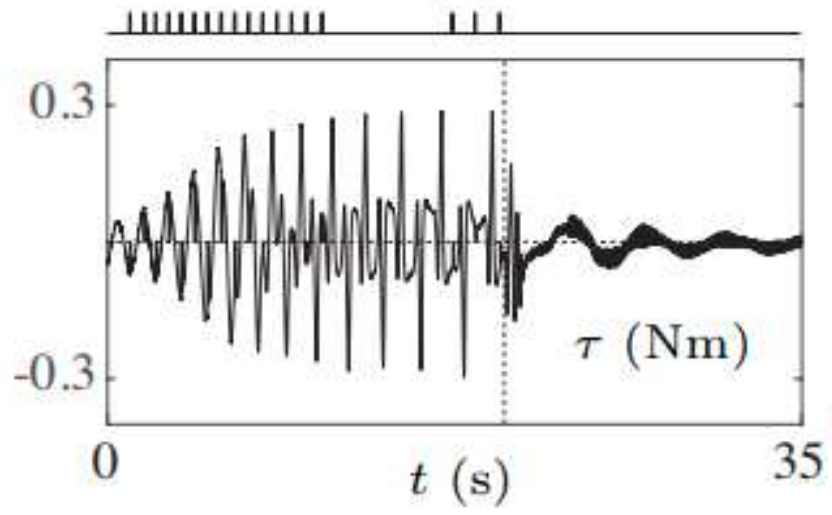

(b)

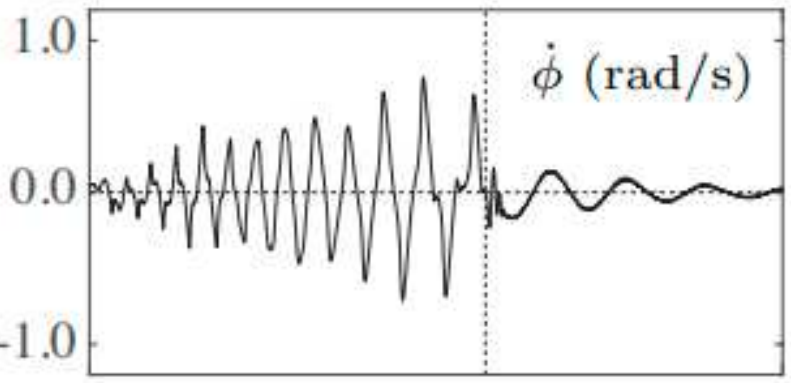

(d)

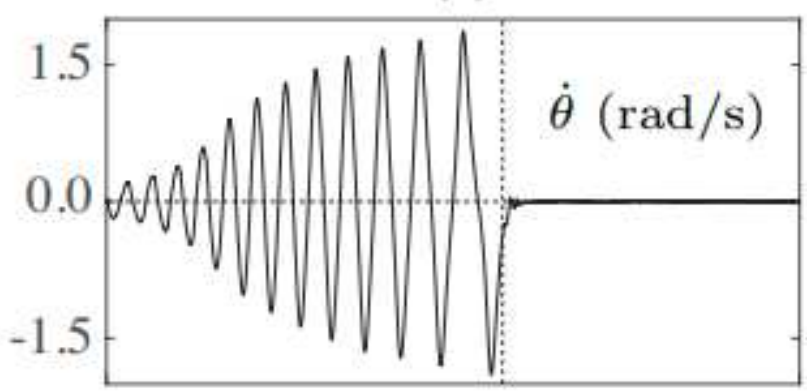

(f)

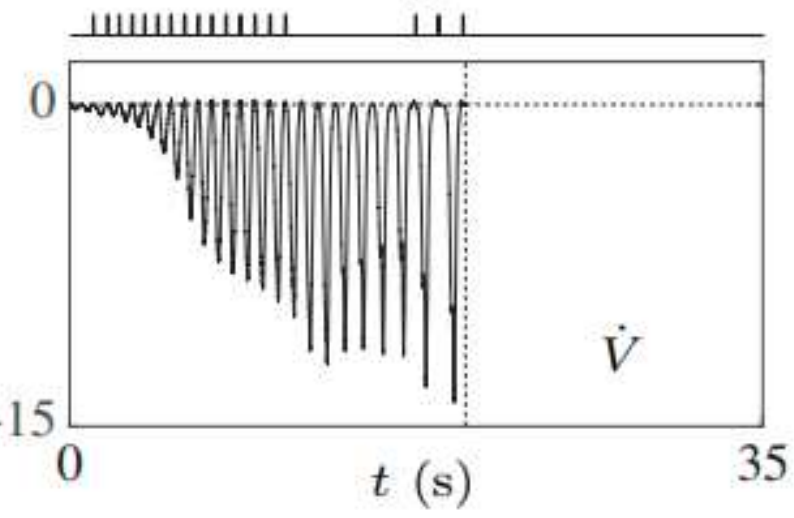

Figure 5

Rotary pendulum experimental results: (a)-(d) are plots of joint angles and joint velocities, (e) control torque, and (f) derivative of Lyapunov-like function. The brake pulses are shown within plots (e) and (f), the peaks represent time intervals when the brakes were engaged. 

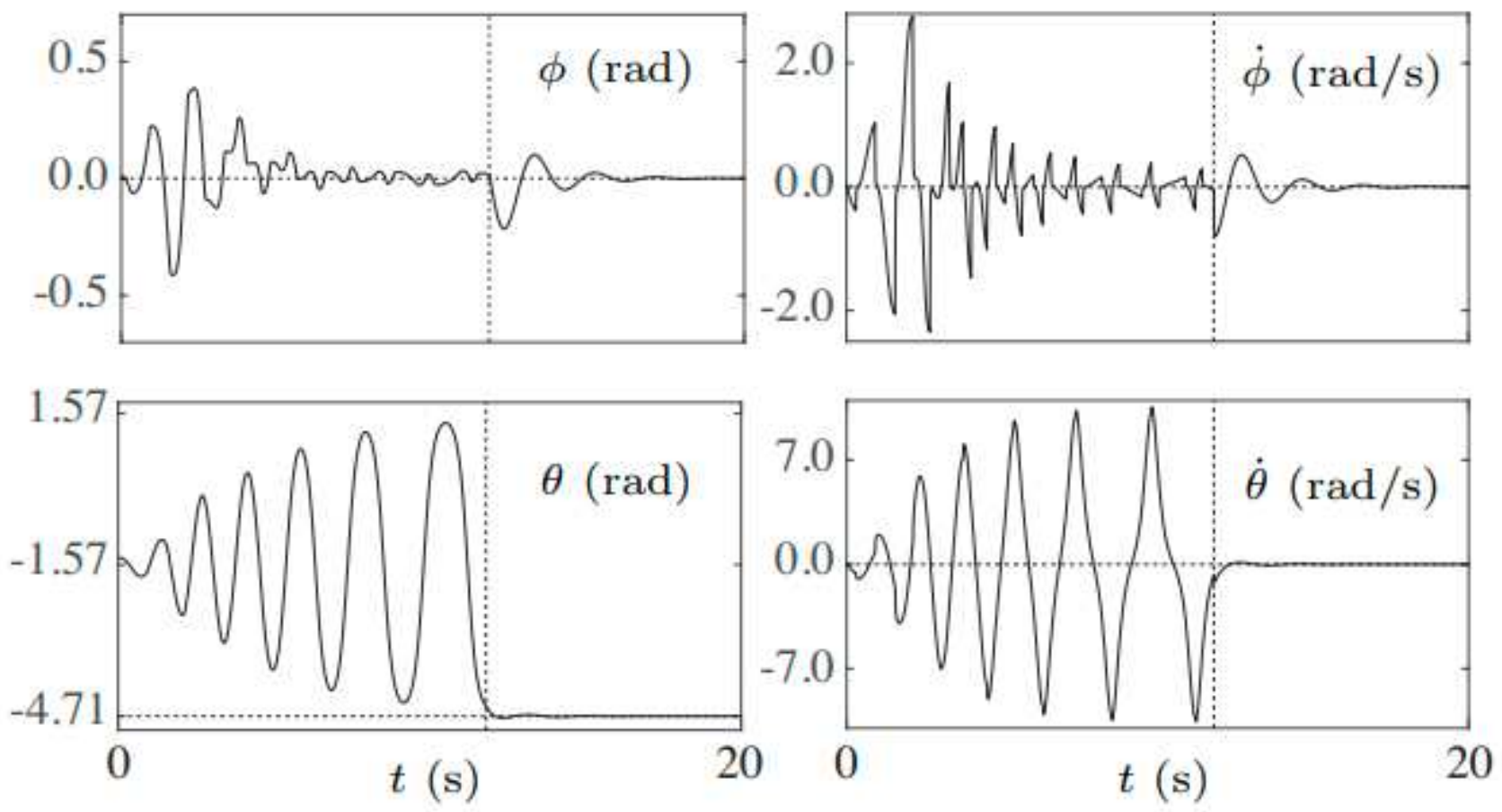

Figure 6

Rotary pendulum simulation results.

\section{Supplementary Files}

This is a list of supplementary files associated with this preprint. Click to download.

- CompressedRP.mp4 